\title{
Discovery of 4-Piperazine Isoquinoline Derivatives as Potent and Brain-Permeable Tau Prion Inhibitors with CDK8 Activity
}

Jean-Marc M. Grandjean ${ }^{\dagger}$, Alexander Y. Jiu ${ }^{\dagger}$, John W. West ${ }^{\dagger}$, Atsushi Aoyagi ${ }^{\ddagger}$, Daniel G. Droege $^{\dagger}$, Manuel Elepano ${ }^{\dagger}$, Makoto Hirasawa ${ }^{\ddagger}$, Masakazu Hirouchi ${ }^{\ddagger}$, Ryo Murakami ${ }^{\ddagger}$, Joanne Lee $^{\dagger}$, Koji Sasaki ${ }^{\ddagger}$, Shimpei Hirano ${ }^{\ddagger}$ Takao Ohyama ${ }^{\star}$, Benjamin C. Tang ${ }^{\dagger}$, Roy J. Vaz ${ }^{\dagger}$, Masahiro Inoue ${ }^{\ddagger}$, Steven H. Olson ${ }^{\dagger}$, Stanley B. Prusiner ${ }^{\dagger}$, Jay Conrad ${ }^{* \dagger}$, and Nick A. Paras ${ }^{\dagger}$

${ }^{\dagger}$ Institute for Neurodegenerative Diseases (IND), UCSF Weill Institute for Neurosciences, University of California, San Francisco, California 94518, United States

¥R\&D Division, Daiichi Sankyo Co., Ltd., 1-2-58 Hiromachi, Shinagawa-ku, Tokyo 140-8710, Japan

\section{Supporting Information}

1. General information for chemistry

2. Experimental procedure for the T24(S) cell assay

3. General procedure for in vivo $K_{\mathrm{p}}$ experiments

4. Procedure for metabolic stability and free fraction assays

5. Free fractions for compounds tested in vivo

6. General procedure for docking and computational experiments

7. Kinome scan data for compound 25

8. Synthetic procedures

9. Compound spectra 


\section{General information for chemistry}

All substrates and reagents were commercially available and used without further purification. Reactions were monitored by LCMS (Agilent Technologies G6100 Series LC/MSD Single Quad) or TLC. Flash chromatography was carried out on a CombiFlash Rf + purification system using RediSep Rf Gold silica gel (20-40 $\mu \mathrm{m})$ purchased from Teledyne Isco, Inc, or using silica gel (200-300 mesh) on Biotage Purification System ${ }^{\circledR}$. Organic solutions were concentrated under reduced pressure on a Heidolph rotary evaporator. ${ }^{1} \mathrm{H}$ and ${ }^{13} \mathrm{C}\left\{{ }^{1} \mathrm{H}\right\}$ NMR spectra were recorded on a Bruker Avance $(400 \mathrm{MHz})$ spectrometer and are internally referenced to residual proton reported as follows: chemical shift $(\delta \mathrm{ppm})$, multiplicity $(s=$ singlet, $d=$ doublet, $\mathrm{t}=$ triplet, $\mathrm{q}=$ quartet, $\mathrm{p}=$ pentet, $\mathrm{dd}=$ doublet of doublets, $\mathrm{ddd}=$ doublet of doublet of doublets, $m=$ multiplet, $b r=$ broad), integration, coupling constant $(\mathrm{Hz})$ and assignment. LC-MS spectra were obtained on Agilent Technologies G6100 series LC/MSD single quad (column: Gemini-nx C18 (4.6x 50 mm, 3.um) or XBRIGE C18 (4.6x 50 mm, 3.5um); Mobile phase: H2O (10 mmol NH4HCO3 ) (A) / ACN(B); Gradient from 10 to $95 \%$ of $B$ in $1.5 \mathrm{~min}$ at $1.8 \mathrm{ml} / \mathrm{min}, 1 \mathrm{ml} / \mathrm{min}, 215 / 254 / 280 \mathrm{~nm}$ ) or (column: Halo 2.7 uM C18 3.0x30mm; mobile phase: $A=$ water (0.1\% TFA) $B=A C N(0.1 \%$ TFA); gradient: 0 min to $0.3 \mathrm{~min}$ held at 5:95 A:B, 0.3 to $3 \mathrm{~min}$ up to 5:95 A:B, $3 \mathrm{~min}$ to $4 \mathrm{~min}$ held at 5:95 A:B, 4 min to $4.1 \mathrm{~min}$ held at 95:5 $A: B, 1 \mathrm{ml} / \mathrm{min}, 215 / 254 / 280 \mathrm{~nm}$ )

Prep-TLC plate or Prep-HPLC were used as indicated in individual procedures. The usual Prep-HPLC method under basic conditions were performed on a Gilson 281(PHG012) using Xtimate C18 $21.2 * 250 \mathrm{~mm} * 10$ um column. The mobile phase was composed of $\mathrm{A}=10 \mathrm{M} \mathrm{NH} \mathrm{HCO}_{3}$ with $0.025 \% \mathrm{NH}_{3} \cdot \mathrm{H}_{2} \mathrm{O}$ and $B=$ Acetonitrile and gradient $58 \% \mathrm{~B}$ for $1 \mathrm{~min}$, then $73 \% \mathrm{~B}$ in $7 \mathrm{~min}$, stop at $15 \mathrm{~min}$. Flow Rate $=30$ $\mathrm{ml} / \mathrm{min}$ and Detective Wavalength $=215 / 254 \mathrm{~nm}$. The usual Prep-HPLC method under acidic conditions was performed on a Teledyne ISCO CombiFlash EZ prep using Luna 5 um C18 (100A) 100x30 mm column. The mobile phase was composed of $A=$ water $(0.1 \%$ TFA $)$ and $B=$ acetonirile $(0.1 \%$ TFA $)$ and gradient 0 to 2 min held at 90:10 A:B then up to 0:100 A:B from 2 mins to 21 mins. Back to 55:45 A:B from 21 mins to $31 \mathrm{mins}$. Flow Rate $=40 \mathrm{ml} / \mathrm{min}$ and detective wavelength $=215 / 254 \mathrm{~nm}$.

Kd values for CDK8 were obtained from Eurofins DiscoverX.

\section{Procedures for the T24(S) cell assay}

A - Cell Line Development.

Sequence encoding full length human ON4R tau containing the P301S mutation fused with EYFP at the Cterminus was synthesized and introduced into the pIRESpuro3 vector (Clontech), were transfected to HEK293T cells (American Type Culture Collection), and monoclonal cell line was generated and maintained as described ${ }^{[1]}$.

B - Phosphotungstic acid precipitation of tau protein in brain samples from Tg2541 mice.

Fresh-frozen brain tissues from 6-7 months old homozygous Tg0N4Rtau*P301S (Tg2541+/+) mice were used to create a $10 \%$ (wt/vol) homogenate in DPBS using the Omni Tissue Homogenizer (Omni International). Phosphotungstic acid (PTA) precipitation of brain samples was performed as described 
(Lee, 2005; Aoyagi, 2019). Briefly, $10 \%$ brain homogenate was incubated in $2 \%$ sarkosyl and $0.5 \%$ benzonase (Sigma) at $37^{\circ} \mathrm{C}$ with constant agitation (1,200 rpm) in an orbital shaker for 2 hours. Sodium PTA was dissolved in double-distilled $\mathrm{H}_{2} \mathrm{O}\left(\mathrm{ddH}_{2} \mathrm{O}\right)$, and the $\mathrm{pH}$ was adjusted to 7.0. PTA was added to the solution to a final concentration of $2 \%$, which was then incubated overnight under the same conditions. The sample was centrifuged at $16,100 \times \mathrm{g}$ for 30 minutes at room temperature, and the supernatant was removed. The resulting pellet was resuspended in $2 \%$ sarkosyl in PBS and $2 \%$ PTA in $d_{d d} \mathrm{H}_{2} \mathrm{O}, \mathrm{pH}$ 7.0. The sample was again incubated for at least 1 hour prior to a second centrifugation. The supernatant was again removed, and the pellet was resuspended in PBS using $10 \%$ of the initial starting volume and stored at $-80^{\circ} \mathrm{C}$.

C - Preparation of cell lysate from monoclonal cells stably expressing tau aggregates.

Monoclonal cells stably expressing tau aggregates were generated by limiting dilution of ON4R tau (P301S)-YFP cells exposed to PTA samples from Tg2541+/+ mouse brains. The presence of a clonal population was confirmed using the IN Cell Analyzer 6000 (GE Healthcare). Lysate of monoclonal cells stably expressing tau aggregates was prepared by harvesting the cells in DPBS containing protease inhibitor (Sigma) using a cell scraper. Cells were placed in sterile conical tubes and snap frozen in liquid $\mathrm{N}_{2}$ for lysing. Tubes were then thawed quickly at $37^{\circ} \mathrm{C}$ and the freeze-thaw cycle was repeated 3 times. Lysate was centrifuged at $500 \times \mathrm{g}$ for $5 \mathrm{~min}$. The supernatant was collected and centrifuged at $1,000 \times \mathrm{g}$ for 5 min. Protein concentration of the supernatant was determined using the bicinchoninic acid assay (Pierce) and stored at $-80^{\circ} \mathrm{C}(\operatorname{Tg} 2541$ cell lysate).

D - Cell Aggregation Assay.

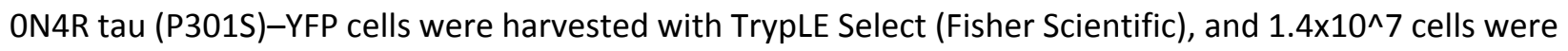
transferred into a $15 \mathrm{ml}$ conical tube. Cells were spun down at $1,000 \mathrm{rpm}$ for $3 \mathrm{~min}$, and the supernatant was removed and cells were resuspended in 100 $\mu$ l Nucleofector solution (Lonza). 300ug of Tg2541 cell lysate was added to cell suspension, and the mixture was transferred into a cuvette and electroporated (Nucleofector, program: Q-001, Lonza). Dilute cells with antibiotic-free DMEM supplemented with $10 \%$ (vol/vol) FBS, and plated in a 384-well plate with black polystyrene walls (Greiner) at a density of 3,000 cells per well with $0.1 \mathrm{\mu g} / \mathrm{ml}$ Hoechst 33342 (Fisher Scientific). After incubation for $2 \mathrm{hr}$ at $37^{\circ} \mathrm{C}$ in a humidified atmosphere of $5 \% \mathrm{CO}_{2}$, compounds were added. Plates were further incubated at $37{ }^{\circ} \mathrm{C}$ for 3 days before imaging on the IN Cell Developer 6000. Images of both the DAPI and FITC channels were collected from four different regions in each well. The images were analyzed using the IN Cell Developer software (GE Healthcare) using an algorithm developed to identify intracellular aggregates only in live cells.

I. S. Lee, J. R. Long, S. B. Prusiner, J. G. Safar, Selective precipitation of prions by polyoxometalate complexes. J. Am. Chem. Soc. 127, 13802-13803 (2005).

(1) - Aoyagi, A.; Condello, C.; J. Stöhr, J.; Yue, W.; Rivera, B., M.; Lee, J., C.; Woerman, A., L.; Halliday, G.; van Duinen, S.; Ingelsson, M.; Lannfelt, L.; Graff, C.; Bird, T., D.; Keene, C., D.; Seeley, W., W.; DeGrado, W., F.; Prusiner, S., B.; $A \beta$ and tau prion-like activities decline with longevity in the Alzheimer's disease human brain Sci. Transl. Med. 2019, 11, eaat8462 


\section{General procedure for in vivo $K_{\mathrm{p}}$ experiments}

To assess brain penetration, we administered compound at $10 \mathrm{mg} / \mathrm{kg}$ in $0.5 \%$ methylcellulose to B6/J mice. Dosing solution is prepared by grinding the compound with slow addition of $0.5 \%$ methylcellulose into a homogeneous suspension. Mice are given compound by oral gavage at $10 \mathrm{uL} / \mathrm{g}$ through a 20 gauge feeding tube. $20 \mathrm{uL}$ of blood is collected at 0.5 hours post-gavage or 0.5 and 1 hour post-gavage or $0.5,1$, and 2 hours post-gavage depending on compound (based on tables 1, 4 and 5 of the main text) into blood collection tubes containing K2EDTA (greiner bio-one). Blood samples are centrifuged to isolate plasma and flash frozen until analysis. Brain is collected at 0.5 hours, 1 hour or 2 hours post-gavage depending on compound (based on tables 1, 4 and 5 of the main text) and flash frozen until analysis. Brain samples are prepared by adding PBS to half the brain at a 1:5 ratio and homogenized (Bertin Precellys 24). Plasma samples are prepared by diluting plasma with PBS at a 1:4 ratio. $15 \mathrm{uL}$ of samples are mixed with $15 \mathrm{uL}$ acetonitrile before addition of $150 \mathrm{uL}$ acetonitrile and methanol (1:1 v/v) containing $0.05 \mathrm{ug} / \mathrm{mL}$ niflumic acid. The mixture is then filtered with Captiva ND plates $(0.2 \mathrm{um})$ into $150 \mathrm{uL}$ water and analyzed with a QTRAP 5500 tandem mass spectrometer (Sciex) coupled with a Nexera X2 series UHPLC (Shimadzu).

\section{Procedure for metabolic stability and free fraction assays}

A - Metabolic stability

Metabolic stability of test compounds was assessed using mouse liver microsomes. Test compounds (1 $\mathrm{uM}$ ) were incubated with mouse liver microsomes (Xenotech Sekisui) at $0.5 \mathrm{mg} / \mathrm{mL}$ in $100 \mathrm{mM}$ potassium phosphate buffer in the presence or absence of a NADPH regenerating system (Promega) at 37C. Aliquots are taken at 0 minutes and 30 minutes and quenched in ice cold methanol:acetonitrile mixture $(1: 1 \mathrm{v} / \mathrm{v})$ containing niflumic acid as an internal standard. Quenched solutions are filtered (Captiva, Agilent) before analysis by LC/MS/MS. Metabolic stability is determined as the analyte:internal standard area ratio of the 30 minute aliquot divided by the 0 minute aliquot. Compound stability is determined using the samples from the absence of NADPH.

\section{B - Protein binding}

Protein binding in cell culture media, brain tissue, and plasma were assessed using rapid equilibrium dialysis. Cell culture media (DMEM supplemented with 10\% FBS and 1\% penicillin/streptomycin), female mouse C57BL/6 brain homogenate (BiolVT, 10\% in PBS), or mouse plasma (BiolVT, 10\% in PBS) spiked with test compound at $5 \mathrm{uM}$ and added to the chamber of the RED device (Thermo Scientific Pierce, 8K MWCO) and isotonic sodium phosphate buffer is added to outer chamber of the RED device and the plate is incubated at 37C. Aliquots of the buffer and matrix are taken at 0 hours and 5 hours and quenched in ice cold methanol:acetonitrile mixture $(1: 1 \mathrm{v} / \mathrm{v})$ containing niflumic acid as an internal standard. Quenched solutions are filtered (Captiva, Agilent) before analysis by LC/MS/MS. 


\section{Free Fractions for compounds tested in vivo}

\begin{tabular}{|c|c|c|c|}
\hline Compound \# & $f_{u, m^{a}}$ & $f_{u, p}^{b}$ & $f_{u, b}{ }^{c}$ \\
\hline 1 & 0.790 & 0.208 & 0.105 \\
\hline 2 & -- & 0.042 & 0.020 \\
\hline 3 & -- & 0.174 & 0.089 \\
\hline 18 & 0.485 & 0.055 & 0.021 \\
\hline 20 & 0.402 & 0.016 & 0.007 \\
\hline 21 & 0.320 & 0.0165 & 0.0055 \\
\hline 25 & 0.510 & 0.015 & 0.001 \\
\hline 26 & 0.507 & 0.0332 & 0.001 \\
\hline 27 & 0.250 & 0.011 & 0.003 \\
\hline 28 & 0.434 & 0.081 & 0.014 \\
\hline
\end{tabular}

\section{General procedure for docking and computational experiments}

All protein and ligand preparation as well as docking was performed using Maestro version 12 available from Schrodinger, LLC, New York, USA.

Based on the structures listed in table 1 (EJMC reference below) ${ }^{[2]}$, the structures with the best resolution from the DMG-in (pdb codes: 5XS2, 5ICP and 4F7S) structures, were used in a cross-docking experiment. All DFG-in ligands listed in the structures in table 1 were extracted from their crystal structure, valences and bond-orders checked and then docked into the protein structures prepared from 5XS2, 5ICP and 4F7A. The resulting lowest energy pose for each ligand was compared with the ligand in the crystal structure from which it was extracted. For 5XS2, only 7 (ligands from 5I5Z, 5HBJ, 5HBE, 5FGK, 5BNJ, 5XQX, 5XS2) of 14 were within 2A RMS. For 5ICP, 10 (ligands from 5IDP, 5IDN, 5HBJ, 5HBH, 5HBE, 5FGK, 5BNJ, 5ICP, 5I5Z, $5 X Q X$ ) out of 14 were within $2 A$ RMS and for 4F7S, only 2 (ligands from 4CRL, 4F7s out of the 14 were within the same cutoff. Therefore, 5ICP was used for subsequent docking experiments. The compounds $28\left(K_{d}=0.77 n M\right), 21\left(K_{d}=30 n M\right), 25\left(K_{d}=32 n M\right), 13\left(K_{d}=600 n M\right)$ (main text, Table 6) when docked using the XP protocol with other values set at their default values, into the 5ICP protein structure yielded lowest energy poses shown in the figure below. In all four structures, the quinoline ring overlaid well on the quinoline ring in the ligand from structure $515 \mathrm{Z}$ (pdb code). In addition, the docked energy, rank ordered the same as compound Kd values with a (lowest Kd, highest docked energy) to $\mathrm{d}$ (highest Kd, lowest docked energy). Similar experiments on the DMG-out structures after cross-docking, did not yield docked structures for all 4 compounds 28, 21, 25 or 13 . 
(2) - Xi, M.; Chen, T.; Wu, C.; Gao, X.; Wu, Y.; Luo, X.; Du, K.; Yu, L.; Cai, T.; Shen, R.; Sun, H. CDK8 as a therapeutic target for cancers and recent developments in discovery of CDK8 inhibitors Eur. J. Med.

Chem. 2019, 164, 77-91.

\section{Kinome scan for compound 25}

Compound 25 was screened at $1 \mu \mathrm{M}$ against 468 kinases using DiscoverX's KINOMEscan ${ }^{\mathrm{TM}}$ screening technology.
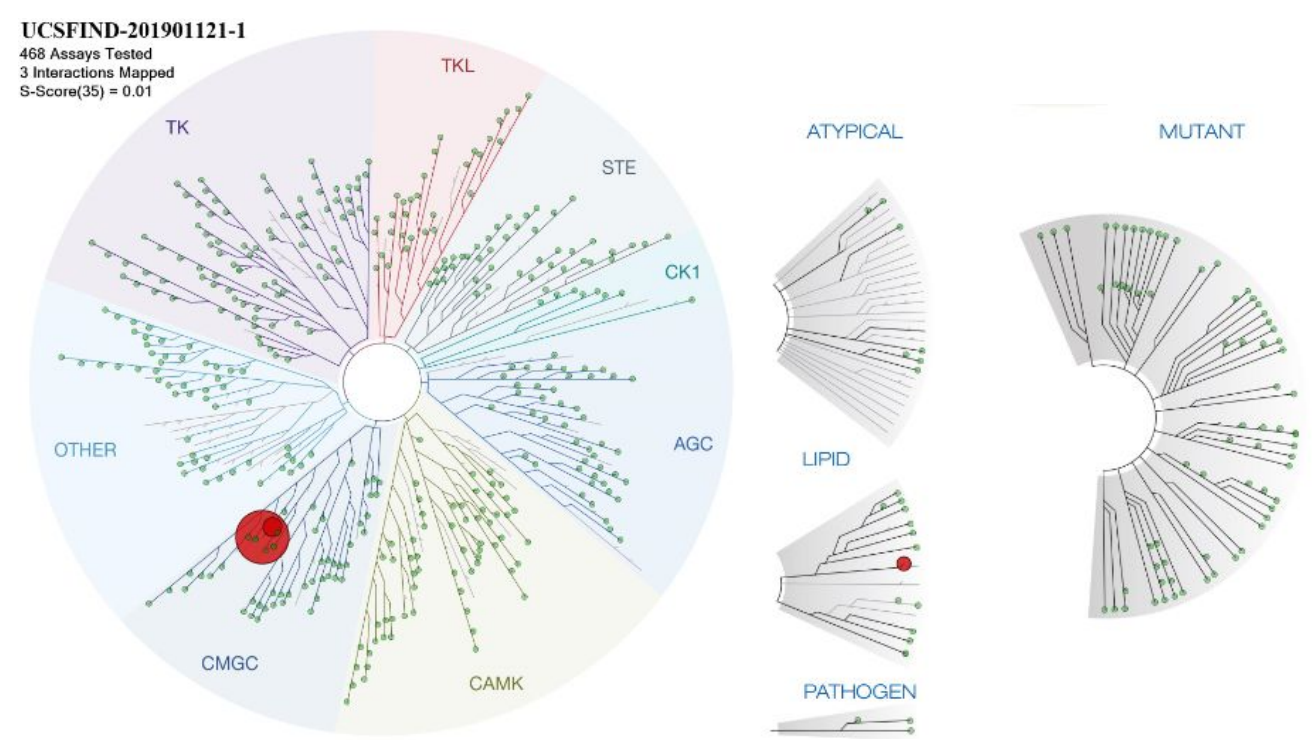

DiscoveRx Gene Symbol

AAK1

ABL1(E255K)-phosphorylated ABL1(F317I)-nonphosphorylated ABL1(F317I)-phosphorylated ABL1(F317L)-nonphosphorylated ABL1(F317L)-phosphorylated ABL1(H396P)-nonphosphorylated ABL1(H396P)-phosphorylated ABL1(M351T)-phosphorylated ABL1(Q252H)-nonphosphorylated ABL1(Q252H)-phosphorylated ABL1(T315I)-nonphosphorylated ABL1(T315I)-phosphorylated ABL1(Y253F)-phosphorylated ABL1-nonphosphorylated ABL1-phosphorylated $A B L 2$ ACVR1
Entrez Gene Symbol AAK1 ABL1 ABL1

ABL1

ABL1

$A B L 1$

ABL1

ABL1

ABL1

ABL1

ABL1

ABL1

ABL1

ABL1

$A B L 1$

ABL1

ABL2

ACVR1
Percent Control

90

100

100

100

100

100

96

95

99

92

100

94

95

97

72

97

97

80 
ACVR1B

ACVR2A

ACVR2B

ACVRL1

ADCK3

ADCK4

AKT1

AKT2

AKT3

ALK

ALK(C1156Y)

ALK(L1196M)

AMPK-alpha1

AMPK-alpha2

ANKK1

ARK5

ASK1

ASK2

AURKA

AURKB

AURKC

AXL

BIKE

BLK

BMPR1A

BMPR1B

BMPR2

$B M X$

BRAF

BRAF(V600E)

BRK

BRSK1

BRSK2

BTK

BUB1

CAMK1

CAMK1B

CAMK1D

CAMK1G

CAMK2A

CAMK2B

CAMK2D

CAMK2G

CAMK4

CAMKK1
ACVR1B 65

ACVR2A $\quad 82$

ACVR2B $\quad 88$

ACVRL1 80

CABC1

ADCK4 97

AKT1 90

AKT2 92

AKT3 95

ALK 100

ALK 89

ALK 100

PRKAA1 100

PRKAA2 93

ANKK1 100

NUAK1 92

MAP3K5 84

MAP3K6 100

AURKA 99

AURKB 100

AURKC $\quad 71$

AXL 90

BMP2K 63

BLK $\quad 76$

BMPR1A 64

BMPR1B 97

BMPR2 92

BMX $\quad 88$

BRAF 100

BRAF 90

PTK6 91

BRSK1 93

BRSK2 73

BTK 98

BUB1 97

CAMK1 94

PNCK 100

CAMK1D 91

CAMK1G 97

CAMK2A 92

CAMK2B 98

CAMK2D 88

CAMK2G

CAMK4 100

CAMKK1 92 


CAMKK2
CASK
CDC2L1
CDC2L2
CDC2L5
CDK11
CDK2
CDK3
CDK4
CDK4-CyclinD1
CDK4-CyclinD3
CDK5
CDK7
CDK8
CDK9
CDKL1
CDKL2
CDKL3
CDKL5
CHEK1
CHEK2
CIT
CLK1
CLK2
CLK3
CLK4
CSF1R
CSF1R-autoinhibited
CSK
CSNK1A1
CSNK1A1L
CSNK1D
CSNK1E
CSNK1G1
CSNK1G2
CSNK1G3
CSNK2A1
CSNK2A2
CTK
DAPK1
DAPK2 3

\begin{tabular}{|c|c|}
\hline CAMKК2 & 77 \\
\hline CASK & 100 \\
\hline CDK11B & 100 \\
\hline $\mathrm{CDC} 2 \mathrm{~L} 2$ & 100 \\
\hline CDK13 & 100 \\
\hline CDK19 & 0.05 \\
\hline CDK2 & 78 \\
\hline CDK3 & 75 \\
\hline CDK4 & 100 \\
\hline CDK4 & 96 \\
\hline CDK4 & 100 \\
\hline CDK5 & 89 \\
\hline CDK7 & 82 \\
\hline CDK8 & 6.3 \\
\hline CDK9 & 99 \\
\hline CDKL1 & 89 \\
\hline CDKL2 & 85 \\
\hline CDKL3 & 75 \\
\hline CDKL5 & 100 \\
\hline CHEK1 & 100 \\
\hline CHEK2 & 100 \\
\hline $\mathrm{CIT}$ & 72 \\
\hline CLK1 & 72 \\
\hline CLK2 & 82 \\
\hline CLK3 & 95 \\
\hline CLK4 & 61 \\
\hline CSF1R & 95 \\
\hline CSF1R & 100 \\
\hline CSK & 99 \\
\hline CSNK1A1 & 92 \\
\hline CSNK1A1L & 100 \\
\hline CSNK1D & 83 \\
\hline CSNK1E & 95 \\
\hline CSNK1G1 & 95 \\
\hline CSNK1G2 & 100 \\
\hline CSNK1G3 & 100 \\
\hline CSNK2A1 & 97 \\
\hline CSNK2A2 & 85 \\
\hline MATK & 91 \\
\hline DAPK1 & 97 \\
\hline DAPK2 & 91 \\
\hline DAPK3 & 98 \\
\hline DCLK1 & 100 \\
\hline DCLK2 & 97 \\
\hline DCLK3 & 10 \\
\hline
\end{tabular}




\begin{tabular}{|c|c|c|}
\hline DDR1 & DDR1 & 100 \\
\hline DDR2 & DDR2 & 94 \\
\hline DLK & MAР3К12 & 100 \\
\hline DMPK & DMPK & 79 \\
\hline DMPK2 & CDC42BPG & 100 \\
\hline DRAK1 & STK17A & 96 \\
\hline DRAK2 & STK17B & 86 \\
\hline DYRK1A & DYRK1A & 100 \\
\hline DYRK1B & DYRK1B & 68 \\
\hline DYRK2 & DYRK2 & 100 \\
\hline EGFR & EGFR & 87 \\
\hline EGFR(E746-A750del) & EGFR & 92 \\
\hline $\operatorname{EGFR}(G 719 C)$ & EGFR & 62 \\
\hline EGFR(G719S) & EGFR & 60 \\
\hline EGFR(L747-E749del, A750P) & EGFR & 89 \\
\hline EGFR(L747-S752del, P753S) & EGFR & 100 \\
\hline EGFR(L747-T751del,Sins) & EGFR & 79 \\
\hline EGFR(L858R) & EGFR & 90 \\
\hline EGFR(L858R,T790M) & EGFR & 97 \\
\hline EGFR(L861Q) & EGFR & 60 \\
\hline EGFR(S752-1759del) & EGFR & 61 \\
\hline EGFR(T790M) & EGFR & 93 \\
\hline EIF2AK1 & EIF2AK1 & 100 \\
\hline EPHA1 & EPHA1 & 100 \\
\hline EPHA2 & EPHA2 & 99 \\
\hline EPHA3 & EPHA3 & 98 \\
\hline EPHA4 & EPHA4 & 100 \\
\hline EPHA5 & EPHA5 & 100 \\
\hline EPHA6 & EPHA6 & 100 \\
\hline EPHA7 & EPHA7 & 93 \\
\hline EPHA8 & EPHA8 & 89 \\
\hline EPHB1 & EPHB1 & 97 \\
\hline EPHB2 & EPHB2 & 73 \\
\hline EPHB3 & EPHB3 & 98 \\
\hline EPHB4 & EPHB4 & 100 \\
\hline EPHB6 & EPHB6 & 100 \\
\hline ERBB2 & ERBB2 & 93 \\
\hline ERBB3 & ERBB3 & 100 \\
\hline ERBB4 & ERBB4 & 91 \\
\hline ERK1 & MAPK3 & 100 \\
\hline ERK2 & MAPK1 & 100 \\
\hline ERK3 & MAPK6 & 99 \\
\hline ERK4 & MAPK4 & 100 \\
\hline ERK5 & MAPK7 & 100 \\
\hline ERK8 & MAPK15 & 96 \\
\hline
\end{tabular}




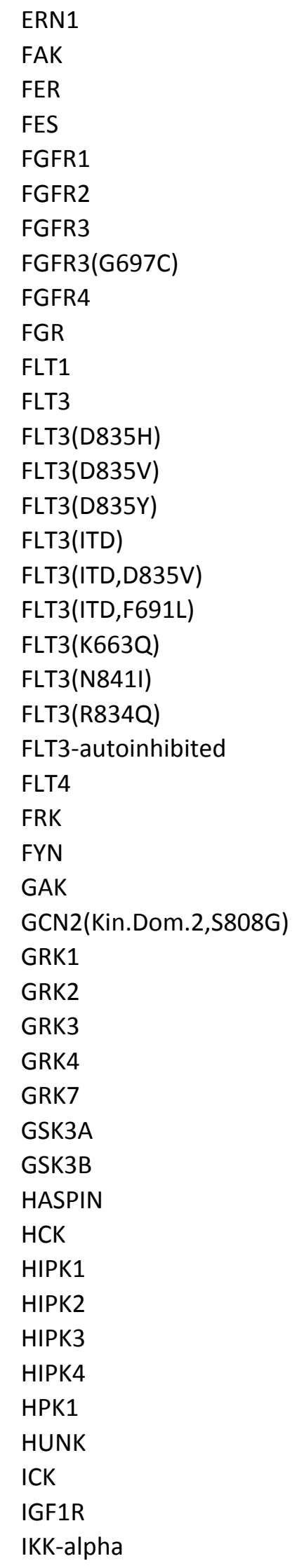

ERN1 100

PTK2 91

FER $\quad 84$

FES 100

FGFR1 82

FGFR2 95

FGFR3 92

FGFR3 97

FGFR4 79

FGR 100

FLT1 71

FLT3 89

FLT3 75

FLT3 99

FLT3 59

FLT3 68

FLT3 100

FLT3 96

FLT3 52

FLT3 100

FLT3 100

FLT3 100

FLT4 85

FRK $\quad 81$

FYN 78

GAK $\quad 87$

EIF2AK4 100

GRK1 100

ADRBK1 90

ADRBK2 100

GRK4 98

GRK7 100

GSK3A 60

GSK3B 100

GSG2 58

HCK 100

HIPK1 68

HIPK2 95

HIPK3 93

HIPK4 71

MAP4K1 100

HUNK 91

ICK 71

IGF1R 97

CHUK 95 


\begin{tabular}{|c|c|c|}
\hline IKK-beta & IKBKB & 88 \\
\hline IKK-epsilon & IKBKE & 92 \\
\hline INSR & INSR & 96 \\
\hline INSRR & INSRR & 100 \\
\hline IRAK1 & IRAK1 & 100 \\
\hline IRAK3 & IRAK3 & 96 \\
\hline IRAK4 & IRAK4 & 100 \\
\hline ITK & ITK & 94 \\
\hline JAK1(JH1domain-catalytic) & JAK1 & 96 \\
\hline JAK1(JH2domain-pseudokinase) & JAK1 & 100 \\
\hline JAK2(JH1domain-catalytic) & JAK2 & 100 \\
\hline JAK3(JH1domain-catalytic) & JAK3 & 97 \\
\hline JNK1 & MAPK8 & 81 \\
\hline JNK2 & MAPK9 & 95 \\
\hline JNK3 & MAPK10 & 86 \\
\hline KIT & KIT & 75 \\
\hline KIT(A829P) & KIT & 100 \\
\hline KIT(D816H) & KIT & 100 \\
\hline KIT(D816V) & KIT & 99 \\
\hline KIT(L576P) & KIT & 85 \\
\hline KIT(V559D) & KIT & 78 \\
\hline KIT(V559D,T670I) & KIT & 80 \\
\hline KIT(V559D,V654A) & KIT & 96 \\
\hline KIT-autoinhibited & KIT & 100 \\
\hline LATS1 & LATS1 & 85 \\
\hline LATS2 & LATS2 & 99 \\
\hline LCK & LCK & 99 \\
\hline LIMK1 & LIMK1 & 87 \\
\hline LIMK2 & LIMK2 & 100 \\
\hline LKB1 & STK11 & 92 \\
\hline LOK & STK10 & 100 \\
\hline LRRK2 & LRRK2 & 100 \\
\hline LRRK2(G2019S) & LRRK2 & 100 \\
\hline LTK & LTK & 99 \\
\hline LYN & LYN & 76 \\
\hline LZK & MAP3K13 & 100 \\
\hline MAK & MAK & 100 \\
\hline MAP3K1 & MAP3K1 & 100 \\
\hline MAP3K15 & MAP3K15 & 100 \\
\hline MAP3К2 & MAP3К2 & 100 \\
\hline МАР3К3 & МАРЗКЗ & 86 \\
\hline MAP3K4 & MAP3K4 & 93 \\
\hline MAP4K2 & MAP4K2 & 88 \\
\hline MAP4K3 & MAP4K3 & 93 \\
\hline MAP4K4 & MAP4K4 & 89 \\
\hline
\end{tabular}




MAP4K5
MAPKAPK2
MAPKAPK5
MARK1
MARK2
MARK3
MARK4
MAST1
MEK1
MEK2
MEK3
MEK4
MEK5
MEK6
MELK
MERTK
MET
MET(M1250T)
MET(Y1235D)
MINK
MKK7
MKNK1
MKNK2
MLCK
MLK1
MLK2
MLK3
MRCKA
MRCKB
MST1
MST1R
MST2
MST3
MST4
MTOR
MUSK
MYLK
MYLK2
MYLK4
MYO3A
MYO3B
NDR1
NDR2
NEK10
MEK1

\begin{tabular}{|c|c|}
\hline MAP4K5 & 94 \\
\hline MAPKAPK2 & 84 \\
\hline MAPKAPK5 & 100 \\
\hline MARK1 & 67 \\
\hline MARK2 & 97 \\
\hline MARK3 & 86 \\
\hline MARK4 & 72 \\
\hline MAST1 & 9 \\
\hline MAP2K1 & 100 \\
\hline MAP2K2 & 100 \\
\hline MAP2K3 & 100 \\
\hline MAP2K4 & 100 \\
\hline MAP2K5 & 68 \\
\hline MAP2K6 & 9 \\
\hline MELK & 76 \\
\hline MERTK & 7 \\
\hline MET & 83 \\
\hline MET & 7 \\
\hline MET & 97 \\
\hline MINK1 & 100 \\
\hline MAP2K7 & 100 \\
\hline MKNK1 & 100 \\
\hline MKNK2 & 100 \\
\hline MYLK3 & 91 \\
\hline МАРЗК9 & 87 \\
\hline MAP3К10 & 72 \\
\hline MAР3К11 & 83 \\
\hline CDC42BPA & 100 \\
\hline CDC42BPB & 99 \\
\hline STK4 & 8 \\
\hline MST1R & 100 \\
\hline STK3 & 100 \\
\hline STK24 & 9 \\
\hline MST4 & 90 \\
\hline MTOR & 9 \\
\hline MUSK & 97 \\
\hline MYLK & 99 \\
\hline MYLK2 & 61 \\
\hline MYLK4 & 92 \\
\hline MYO3A & 8 \\
\hline MYO3B & 57 \\
\hline STK38 & 100 \\
\hline STK38L & 9 \\
\hline NEK1 & 100 \\
\hline NEK10 & 10 \\
\hline
\end{tabular}




NEK11
NEK2
NEK3
NEK4
NEK5
NEK6
NEK7
NEK9
NIK
NIM1
NLK
OSR1
p38-alpha
p38-beta
p38-delta
p38-gamma
PAK1
PAK2
PAK3
PAK4
PAK6
PAK7
PCTK1
PCTK2
PCTK3
PDGFRA
PDGFRB
PDPK1
PFCDPK1(P.falciparum)
PFPK5(P.falciparum)
PFTAIRE2
PFTK1
PHKG1
PHKG2
PIK3C2B
PIK3C2G
PIK3CA
PIK3CA(C420R)
PIK3CA(E542K)
PIK3CA(E545A)
PIK3CA(E545K)
PIK3CA(H1047L)
PIK3CA(H1047Y)
PIK3CA(I800L)
PIK3CA(M1043I)

NEK11 100

NEK2 91

NEK3 87

NEK4 99

NEK5 98

NEK6 100

NEK7 98

NEK9 98

MAP3K14 97

MGC42105 93

NLK 83

OXSR1 100

MAPK14 100

MAPK11 76

MAPK13 100

MAPK12 96

PAK1 100

PAK2 100

PAK3 97

PAK4 100

PAK6 100

PAK7 96

CDK16 100

CDK17 100

CDK18 98

PDGFRA 95

PDGFRB 99

PDPK1 74

CDPK1 91

MAL13P1.279 94

CDK15 96

CDK14 100

PHKG1 99

PHKG2 89

PIK3C2B 100

PIK3C2G 89

PIK3CA 98

PIK3CA 92

PIK3CA 92

PIK3CA 92

PIK3CA 87

PIK3CA 100

PIK3CA 99

PIK3CA 95

PIK3CA 97 


PIK3CA(Q546K)
PIK3CB
PIK3CD
PIK3CG
PIK4CB
PIKFYVE
PIM1
PIM2
PIM3
PIP5K1A
PIP5K1C
PIP5K2B
PIP5K2C
PKAC-alpha
PKAC-beta
PKMYT1
PKN1
PKN2
PKNB(M.tuberculosis)
PLK1
PLK2
PLK3
PLK4
PRKCD
PRKCE
PRKCH
PRKCI
PRKCQ
PRKD1
PRKD2
PRKD3
PRKG1
PRKG2
PRKR
PRKX
PRP4
PYK2
QSK
RAF1
RET
RET(M918T)
RET(V804L)
RET(V804M)
RIOK1
RIOK2

PIK3CA 93

PIK3CB 97

PIK3CD 100

PIK3CG 84

PI4KB 15

PIKFYVE 79

PIM1 95

PIM2 89

PIM3 95

PIP5K1A 74

PIP5K1C 87

PIP4K2B 76

PIP4K2C 66

PRKACA 91

PRKACB 76

PKMYT1 72

PKN1 100

PKN2 88

pknB 99

PLK1 100

PLK2 89

PLK3 95

PLK4 89

PRKCD 79

PRKCE 95

$\mathrm{PRKCH}$

$\mathrm{PRKCl} \quad 96$

PRKCQ 100

PRKD1 100

PRKD2 98

PRKD3 100

PRKG1 82

PRKG2 99

EIF2AK2 100

PRKX 92

PRPF4B 97

PTK2B 97

KIAA0999 88

RAF1 94

RET $\quad 82$

RET $\quad 78$

RET $\quad 79$

RET $\quad 89$

RIOK1 56

RIOK2 91 


\begin{tabular}{|c|c|c|}
\hline RIOK3 & RIOK3 & 74 \\
\hline RIPK1 & RIPK1 & 100 \\
\hline RIPK2 & RIPK2 & 70 \\
\hline RIPK4 & RIPK4 & 99 \\
\hline RIPK5 & DSTYK & 87 \\
\hline ROCK1 & ROCK1 & 100 \\
\hline ROCK2 & ROCK2 & 100 \\
\hline ROS1 & ROS1 & 82 \\
\hline RPS6KA4(Kin.Dom.1-N-terminal) & RPS6KA4 & 74 \\
\hline RPS6KA4(Kin.Dom.2-C-terminal) & RPS6KA4 & 100 \\
\hline RPS6KA5(Kin.Dom.1-N-terminal) & RPS6KA5 & 85 \\
\hline RPS6KA5(Kin.Dom.2-C-terminal) & RPS6KA5 & 97 \\
\hline RSK1(Kin.Dom.1-N-terminal) & RPS6KA1 & 97 \\
\hline RSK1(Kin.Dom.2-C-terminal) & RPS6KA1 & 100 \\
\hline RSK2(Kin.Dom.1-N-terminal) & RPS6KA3 & 96 \\
\hline RSK2(Kin.Dom.2-C-terminal) & RPS6KA3 & 81 \\
\hline RSK3(Kin.Dom.1-N-terminal) & RPS6KA2 & 97 \\
\hline RSK3(Kin.Dom.2-C-terminal) & RPS6KA2 & 90 \\
\hline RSK4(Kin.Dom.1-N-terminal) & RPS6KA6 & 100 \\
\hline RSK4(Kin.Dom.2-C-terminal) & RPS6KA6 & 83 \\
\hline S6K1 & RPS6KB1 & 100 \\
\hline SBK1 & SBK1 & 100 \\
\hline SGK & SGK1 & 100 \\
\hline SgK110 & SgK110 & 85 \\
\hline SGK2 & SGK2 & 100 \\
\hline SGK3 & SGK3 & 99 \\
\hline SIK & SIK1 & 100 \\
\hline SIK2 & SIK2 & 92 \\
\hline SLK & SLK & 97 \\
\hline SNARK & NUAK2 & 100 \\
\hline SNRK & SNRK & 100 \\
\hline SRC & SRC & 93 \\
\hline SRMS & SRMS & 100 \\
\hline SRPK1 & SRPK1 & 75 \\
\hline SRPK2 & SRPK2 & 100 \\
\hline SRPK3 & SRPK3 & 86 \\
\hline STK16 & STK16 & 86 \\
\hline STK33 & STK33 & 61 \\
\hline STK35 & STK35 & 91 \\
\hline STK36 & STK36 & 91 \\
\hline STK39 & STK39 & 73 \\
\hline SYK & SYK & 86 \\
\hline TAK1 & MAP3K7 & 98 \\
\hline TAOK1 & TAOK1 & 100 \\
\hline TAOK2 & TAOK2 & 100 \\
\hline
\end{tabular}




\begin{tabular}{|c|c|c|}
\hline TAOK3 & TAOK3 & 100 \\
\hline TBK1 & TBK1 & 100 \\
\hline TEC & TEC & 87 \\
\hline TESK1 & TESK1 & 93 \\
\hline TGFBR1 & TGFBR1 & 96 \\
\hline TGFBR2 & TGFBR2 & 82 \\
\hline TIE1 & TIE1 & 100 \\
\hline TIE2 & TEK & 67 \\
\hline TLK1 & TLK1 & 92 \\
\hline TLK2 & TLK2 & 100 \\
\hline TNIK & TNIK & 100 \\
\hline TNK1 & TNK1 & 89 \\
\hline TNK2 & TNK2 & 91 \\
\hline TNNI3K & TNNI3K & 79 \\
\hline TRKA & NTRK1 & 100 \\
\hline TRKB & NTRK2 & 100 \\
\hline TRKC & NTRK3 & 100 \\
\hline TRPM6 & TRPM6 & 100 \\
\hline TSSK1B & TSSK1B & 100 \\
\hline TSSK3 & TSSK3 & 81 \\
\hline TTK & TTK & 83 \\
\hline TXK & TXK & 93 \\
\hline TYK2(JH1domain-catalytic) & TYK2 & 88 \\
\hline TYK2(JH2domain-pseudokinase) & TYK2 & 100 \\
\hline TYRO3 & TYRO3 & 88 \\
\hline ULK1 & ULK1 & 100 \\
\hline ULK2 & ULK2 & 90 \\
\hline ULK3 & ULK3 & 100 \\
\hline VEGFR2 & KDR & 100 \\
\hline VPS34 & PIK3C3 & 92 \\
\hline VRK2 & VRK2 & 100 \\
\hline WEE1 & WEE1 & 85 \\
\hline WEE2 & WEE2 & 95 \\
\hline WNK1 & WNK1 & 99 \\
\hline WNK2 & WNK2 & 100 \\
\hline WNK3 & WNK3 & 83 \\
\hline WNK4 & WNK4 & 94 \\
\hline YANK1 & STK32A & 100 \\
\hline YANK2 & STK32B & 97 \\
\hline YANK3 & STK32C & 99 \\
\hline YES & YES1 & 89 \\
\hline YSK1 & STK25 & 100 \\
\hline YSK4 & MAP3K19 & 100 \\
\hline ZAK & ZAK & 94 \\
\hline ZAP70 & ZAP70 & 96 \\
\hline
\end{tabular}




\section{Synthetic procedures}

General synthetic scheme for the synthesis of compounds 1 to 15

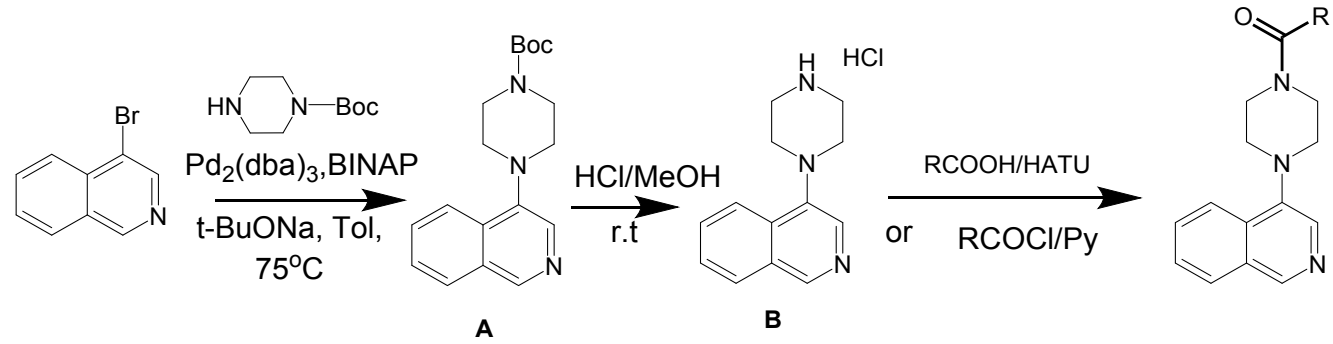

A - tert-butyl 4-(isoquinolin-4-yl)piperazine-1-carboxylate. Under N2, to a solution of 4bromoisoquinoline $(10 \mathrm{~g}, 48.06 \mathrm{mmol})$, tert-butyl piperazine-1-carboxylate $(18 \mathrm{~g}, 96.13 \mathrm{mmol})$, t-BuONa $(14 \mathrm{~g}, 144.19 \mathrm{mmol})$ in anhydrous toluene $(80 \mathrm{~mL})$ was added $\mathrm{Pd} 2(\mathrm{dba}) 3(440 \mathrm{mg}, 0.480 \mathrm{mmol})$ and BINAP(600mg, $0.961 \mathrm{mmol})$. The mixture was purged with N2 and stirred at $75^{\circ} \mathrm{C}$ for $20 \mathrm{hrs}$. The solvent was removed under reduced pressure, $\mathrm{H} 2 \mathrm{O}(250 \mathrm{~mL})$ was added and extracted with ethyl acetate $(250 \mathrm{~mL}$ $\mathrm{x} 3$ ). The organic layer was collected, dried with anhydrous $\mathrm{Na} 2 \mathrm{SO} 4$ and concentrated to afford the crude product which was purified by column chromatography (silica gel, Ethyl Acetate : Petroleum Ether $=36: 64$ ) to afford tert-butyl 4-(isoquinolin-4-yl)piperazine-1-carboxylate $\mathbf{A}(13.5 \mathrm{~g}, 90 \%$ yield, 100 purity) as a yellow solid. LC/MS C ${ }_{18} \mathrm{H}_{23} \mathrm{~N}_{3} \mathrm{O}_{2}[\mathrm{M}+\mathrm{H}]^{+}$calculated $m / z=314.18$; found $m / z=314.0 ; 1.98$ mins at $\lambda=254$ $\mathrm{nm}$.

B - 4-(piperazin-1-yl) isoquinoline hydrochloride. At $25^{\circ} \mathrm{C}$, to a flask containing tert-butyl 4-(isoquinolin4-yl) piperazine-1-carboxylate $\mathbf{A}(3 \mathrm{~g}, 9.58 \mathrm{mmol})$ was added $\mathrm{HCl}$ in $\mathrm{MeOH}$ solution $(3 \mathrm{M}, 25 \mathrm{~mL})$. The reaction was sealed and stirred at room temperature overnight. The precipitate was collected by filtration and dried under vacuum to afford 4-(piperazin-1-yl) isoquinoline hydrochloride B $(2.45 \mathrm{~g}, 99.9 \%$ yield, $100 \%$ purity) as a yellow solid. LC/MS $\mathrm{C}_{23} \mathrm{H}_{15} \mathrm{~N}_{3}[\mathrm{M}+\mathrm{H}]^{+}$calculated $m / z=213.13 .18$; found $\mathrm{m} / \mathrm{z}=214.0$; 1.49 mins at $\lambda=254 \mathrm{~nm}$

1 - $\mathbf{N}$-\{2-[4-(isoquinolin-4-yl)piperazin-1-yl]-2-oxoethyl\}-N-methylthiophene-2-carboxamide. 1-[4(isoquinolin-4-yl)piperazin-1-yl]-2-(methylamino)ethan-1-one hydrochloride $(0.1 \mathrm{~g}, 0.94 \mathrm{mmol})$, thiophene-2-carboxylic acid (119 mg, $0.94 \mathrm{mmol})$, triethylamine $(207 \mathrm{mg}, 2.05 \mathrm{mmol})$, and HATU (387 mg, $1.02 \mathrm{mmol}$ ) were dissolved in N,N-dimethylformamide $(5 \mathrm{~mL})$ and stirred at room temperature overnight. The reaction mixture was diluted with saturated sodium bicarbonate and the product was extracted with dichloromethane. The product was dry loaded onto silica gel and purified by column chromatography (silica gel, $\mathrm{CH} 2 \mathrm{Cl} 2$ : $\mathrm{MeOH}=9: 1$ ) yielding $\mathrm{N}$-(2-(4-(isoquinolin-4-yl)piperazin-1-yl)-2-oxoethyl)- $\mathrm{N}$ methylthiophene-2-carboxamide $\left(65 . .0 \mathrm{mg}, 53.2 \%, 99 \%\right.$ purity) as a white solid. LC/MS $[\mathrm{M}+\mathrm{H}]^{+} \mathrm{m} / \mathrm{z}=$ 395.0; 1.64 mins at $\lambda=254 \mathrm{~nm}$. ${ }^{1} \mathrm{H}-\mathrm{NMR}$ (DMSO, $\left.400 \mathrm{MHz}\right) \delta(\mathrm{ppm})=9.06(\mathrm{~s}, 1 \mathrm{H}), 8.09-8.25(\mathrm{~m}, 3 \mathrm{H})$, 7.75 - 7.87 (m, 2H), 7.66 - 7.75 (m, 1H), 7.16 (br. s., $1 \mathrm{H}), 5.76$ (s, 3H), 4.44 (br.s., $1 \mathrm{H}), 3.78$ (br. s., $2 \mathrm{H}), 3.26$ 
(br. s., 2H), 3.11 (br. s., 5H), 3.01 (br. s., $1 \mathrm{H}) .{ }^{13} \mathrm{C}-\mathrm{NMR}\left(\mathrm{CDCl}_{3}, 100 \mathrm{MHz}\right) \delta(\mathrm{ppm})=166.5,147.8,143.2$, $137.1,131.7,130.8,129.2,128.4,127.8,126.9,122.3,52.7,52.5,45.4,42.4$. HRMS (ESI) $\mathrm{C}_{21} \mathrm{H}_{22} \mathrm{~N}_{4} \mathrm{O}_{2} \mathrm{~S}$ [M $+\mathrm{Na}]^{+}$calculated $m / z=417.1361 ;$ found $m / z=417.1350$

2 - N-methyl-N-(2-(4-(naphthalen-1-yl) piperazin-1-yl)-2-oxoethyl) thiophene-2-carboxamide. To a solution of 2-(N-methylthiophene-2-carboxamido) acetic acid ( $80 \mathrm{mg}, 0.40 \mathrm{mmol}), 1$-(naphthalen-1-yl) piperazine (102 mg, $0.48 \mathrm{mmol}$ ) and HATU (183 mg, $0.48 \mathrm{mmol}$ ) in DMF (3 mL) was added DIEA (1.5 mL) at room temperature.The reaction was stirred at room temperature for one hour. The reaction mixture was directly purified by Prep-HPLC under basic conditions to afford N-methyl-N-(2-(4-(naphthalen-1-yl) piperazin-1-yl)-2-oxoethyl) thiophene-2-carboxamide (60 mg, 38\% yield, $100 \%$ purity) as a white solid. ${ }^{1} \mathrm{H}$ NMR $(400 \mathrm{MHz}, \mathrm{CDCl}) \delta(\mathrm{ppm})=8.20(\mathrm{~d}, \mathrm{~J}=8.9 \mathrm{~Hz}, 1 \mathrm{H}), 7.88-7.80(\mathrm{~m}, 1 \mathrm{H}), 7.60(\mathrm{~d}, J=8.2 \mathrm{~Hz}, 1 \mathrm{H}), 7.55$ $-7.45(\mathrm{~m}, 4 \mathrm{H}), 7.41(\mathrm{t}, J=7.8 \mathrm{~Hz}, 1 \mathrm{H}), 7.08(\mathrm{~d}, J=7.4 \mathrm{~Hz}, 2 \mathrm{H}), 4.43(\mathrm{~s}, 2 \mathrm{H}), 3.76(\mathrm{br}, 3 \mathrm{H}), 3.26(\mathrm{br}, 8 \mathrm{H}) .{ }^{13} \mathrm{C}-$ $\operatorname{NMR}\left(\mathrm{CDCl}_{3}, 100 \mathrm{MHz}\right) \delta(\mathrm{ppm})=176.7,148.0,132.2,131.6,130.3,128.3,127.5,122.4,52.8,45.5,38.8$, 28.5. HRMS (ESI) $\mathrm{C}_{18} \mathrm{H}_{23} \mathrm{~N}_{3} \mathrm{O}[\mathrm{M}+\mathrm{Na}]^{+}$calculated $\mathrm{m} / z=320.1739$; found $\mathrm{m} / z=320.1739$

3 - 1-(4-(isoquinolin-4-yl) piperazin-1-yl)-2,2-dimethylpropan-1-one. At $0^{\circ} \mathrm{C}$, to a solution of 4-(piperazin1-yl) isoquinoline hydrochloride $\mathbf{B}(60 \mathrm{mg}, 0.24 \mathrm{mmol})$ in Pyridine $(3 \mathrm{~mL})$ was added the pivaloyl chloride (50.4 $\mathrm{mg}, 0.42 \mathrm{mmol}$ ). The reaction was stirred at room temperature for $2 \mathrm{hrs}$. The solvent was removed under reduced pressure and the crude product was purified via Prep-HPLC under basic conditions to afford 1-(4-(isoquinolin-4-yl) piperazin-1-yl)-2,2-dimethylpropan-1-one (25 mg, 35\% yield, 100\% purity) as a yellow solid. ${ }^{1} \mathrm{H}-\mathrm{NMR}(500 \mathrm{MHz}, \mathrm{CDCl} 3) \delta(\mathrm{ppm})=9.02(\mathrm{~s}, 1 \mathrm{H}), 8.19(\mathrm{~s}, 1 \mathrm{H}), 8.13(\mathrm{~d}, J=8.5 \mathrm{~Hz}, 1 \mathrm{H}), 7.99$ (d, J=8.2 Hz, 1H), $7.74(\mathrm{t}, J=7.6 \mathrm{~Hz}, 1 \mathrm{H}), 7.63(\mathrm{t}, J=7.5 \mathrm{~Hz}, 1 \mathrm{H}), 3.94(\mathrm{br}, 4 \mathrm{H}), 3.18(\mathrm{br}, 4 \mathrm{H}), 1.35(\mathrm{~s}, 9 \mathrm{H})$. ${ }^{13} \mathrm{C}-\mathrm{NMR}\left(\mathrm{CDCl}_{3}, 100 \mathrm{MHz}\right) \delta(\mathrm{ppm})=166.4,148.7,137.3,134.8,128.7,128.6,126.8,126.1,125.8,124.4$, 123.1, 115.1, 53.1, 52.9, 45.5, 42.6. . HRMS (ESI) $\mathrm{C}_{22} \mathrm{H}_{23} \mathrm{~N}_{3} \mathrm{O}_{2} \mathrm{~S}[\mathrm{M}+\mathrm{Na}]^{+}$calculated $\mathrm{m} / \mathrm{z}=416.1409$; found $m / z=416.1404$

4 - 4-[4-(3-methyloxetane-3-carbonyl)piperazin-1-yl]isoquinoline. 4-(piperazin-1-yl)isoquinoline hydrochloride $(50 \mathrm{mg}, 0.200 \mathrm{mmol})$, triethylamine $(0.0836 \mathrm{~mL}, 0.600 \mathrm{mmol})$, HATU $(76.0 \mathrm{mg}, 0.200 \mathrm{mmol})$ and 3-methyloxetane-3-carboxylic acid $(23.2 \mathrm{mg}, 0.200 \mathrm{mmol})$ were stirred in DMF $(2 \mathrm{~mL})$ under inert atmosphere. After 12 hours the reaction was quenched with brine and extracted three times with ether. The ethereal phase was washed three times with brine. The solvent was removed and the crude was purified by column chromatography (silica gel, $\mathrm{CH} 2 \mathrm{Cl} 2$ : $\mathrm{MeOH}=95: 5)$. 4-[4-(3-methyloxetane-3carbonyl)piperazin-1-yl]isoquinoline recovered as a white solid (25 mg, 40\% yield, 98\% purity). LC/MS $\mathrm{C}_{18} \mathrm{H}_{21} \mathrm{~N}_{3} \mathrm{O}_{2}[\mathrm{M}+\mathrm{H}]^{+}$calculated $\mathrm{m} / \mathrm{z}=312.16$; found $\mathrm{m} / \mathrm{z}=312.2 ; 1.47$ mins at $\lambda=254 \mathrm{~nm} .{ }^{1} \mathrm{H}-\mathrm{NMR}$ (DMSO, $400 \mathrm{MHz}) \delta(\mathrm{ppm})=9.04(\mathrm{~s}, 1 \mathrm{H}), 8.21(\mathrm{~s}, 1 \mathrm{H}), 8.13(\mathrm{~d}, 2 \mathrm{H}, \mathrm{J}=8.8 \mathrm{~Hz}), 8.00(\mathrm{dd}, 1 \mathrm{H}, \mathrm{J} 1=\mathrm{J} 2=7.2 \mathrm{~Hz}), 7.76$ $(\mathrm{dd}, 1 \mathrm{H}, \mathrm{J} 1=\mathrm{J} 2=7.6 \mathrm{~Hz}), 7.65(\mathrm{dd}, 1 \mathrm{H}, \mathrm{J} 1=\mathrm{J} 2=7.6 \mathrm{~Hz}), 4.11-3.89(\mathrm{~m}, 8 \mathrm{H}) 3.38-3.31(\mathrm{~m}, 1 \mathrm{H}), 3.22-3.18(\mathrm{~m}$, $4 \mathrm{H}), 2.37-2.29(\mathrm{~m}, 1 \mathrm{H}), 2.19-2.15(\mathrm{~m}, 1 \mathrm{H})$.

5 - 4-[4-(oxolane-3-carbonyl)piperazin-1-yl]isoquinoline. 4-(piperazin-1-yl)isoquinoline hydrochloride ( $50 \mathrm{mg}, 0.200 \mathrm{mmol})$, triethylamine $(0.0836 \mathrm{~mL}, 0.600 \mathrm{mmol})$, HATU $(76.0 \mathrm{mg}, 0.200 \mathrm{mmol}$ ) and oxolane-3carboxylic acid $(23.0 \mathrm{mg}, 0.200 \mathrm{mmol}$ ) were stirred in DMF $(2 \mathrm{~mL})$ under inert atmosphere. After 12 hours the reaction was quenched with brine and extracted three times with ether. The ethereal phase was washed three times with brine. The solvent was removed and the crude was purified by column 
chromatography (silica gel, $\mathrm{CH} 2 \mathrm{Cl} 2$ : $\mathrm{MeOH}=95: 5$ ). 4-[4-(oxolane-3-carbonyl)piperazin-1-yl]isoquinoline recovered as a white solid ( $25 \mathrm{mg}, 40 \%$ yield, 98\% purity). $\mathrm{LC} / \mathrm{MS} \mathrm{C}_{18} \mathrm{H}_{21} \mathrm{~N}_{3} \mathrm{O}_{2}[\mathrm{M}+\mathrm{H}]^{+}$calculated $\mathrm{m} / z=$ 312.16; found $m / z=312.2 ; 1.50$ mins at $\lambda=254 \mathrm{~nm}$. ${ }^{1} \mathrm{H}-\mathrm{NMR}$ (DMSO, $\left.400 \mathrm{MHz}\right) \delta(\mathrm{ppm})=9.10(\mathrm{~s}, 1 \mathrm{H})$, $8.24(\mathrm{~s}, 1 \mathrm{H}), 8.16(\mathrm{~d}, 2 \mathrm{H}, \mathrm{J}=8.8 \mathrm{~Hz}$ ), $7.85(\mathrm{dd}, 1 \mathrm{H}, \mathrm{J} 1=\mathrm{J} 2=7.2 \mathrm{~Hz}), 7.73(\mathrm{dd}, 1 \mathrm{H}, \mathrm{J} 1=\mathrm{J} 2=7.6 \mathrm{~Hz}$ ), 4.89 (d, $2 \mathrm{H}, \mathrm{J}=6.0 \mathrm{~Hz}$ ), $4.35(\mathrm{~d}, 2 \mathrm{H}, \mathrm{J}=6.0 \mathrm{~Hz}) 3.80(\mathrm{bs}, 2 \mathrm{H}), 3.34(\mathrm{bs}, 2 \mathrm{H}), 3.13(\mathrm{bs}, 4 \mathrm{H}), 1.64(\mathrm{~s}, 3 \mathrm{H})$.

6 - 1-(4-(isoquinolin-4-yl) piperazin-1-yl)-2-(tetrahydrofuran-2-yl)ethanone. To a solution of 2(tetrahydrofuran-2-yl) acetic acid $(50 \mathrm{mg}, 0.38 \mathrm{mmol}), 4-($ piperazin-1-yl)isoquinoline hydrochloride (100 $\mathrm{mg}, 0.38 \mathrm{mmol})$ and HATU $(152 \mathrm{mg}, 0.38 \mathrm{mmol})$ in DMF $(3 \mathrm{~mL})$ was added DIEA $(1.5 \mathrm{~mL})$ at room temperature. The reaction mixture was directly purified by Prep-HPLC under basic conditions to afford 1(4-(isoquinolin-4-yl) piperazin-1-yl)-2-(tetrahydrofuran-2-yl)ethanone (64mg, 52\% yield, 99\% purity) as a white solid. LC/MS $\mathrm{C}_{19} \mathrm{H}_{23} \mathrm{~N}_{3} \mathrm{O}_{2}[\mathrm{M}+\mathrm{H}]^{+}$calculated $\mathrm{m} / z=326.18$; found $\mathrm{m} / \mathrm{z}=326.1 ; 1.73$ mins at $\lambda=254$ nm. ${ }^{1} \mathrm{H}-\mathrm{NMR}\left(400 \mathrm{MHz}, \mathrm{CDCl}_{3}\right) \delta(\mathrm{ppm})=9.02(\mathrm{~s}, 1 \mathrm{H}), 8.19(\mathrm{~s}, 1 \mathrm{H}), 8.12(\mathrm{~d}, J=8.4 \mathrm{~Hz}, 1 \mathrm{H}), 7.98(\mathrm{~d}, J=8.1$ $\mathrm{Hz}, 1 \mathrm{H}), 7.74(\mathrm{dd}, J=11.2,4.1 \mathrm{~Hz}, 1 \mathrm{H}), 7.62(\mathrm{t}, J=7.5 \mathrm{~Hz}, 1 \mathrm{H}), 4.39-4.19(\mathrm{~m}, 1 \mathrm{H}), 3.83(\mathrm{dq}, J=15.3,7.0$ $\mathrm{Hz}, 6 \mathrm{H}), 3.31-3.00(\mathrm{~m}, 4 \mathrm{H}), 2.80(\mathrm{dd}, J=14.9,6.5 \mathrm{~Hz}, 1 \mathrm{H}), 2.56(\mathrm{dd}, J=14.9,6.0 \mathrm{~Hz}, 1 \mathrm{H}), 2.18(\mathrm{dt}, J=12.6$, $6.4 \mathrm{~Hz}, 1 \mathrm{H}), 2.01-1.86(\mathrm{~m}, 2 \mathrm{H}), 1.66-1.56(\mathrm{~m}, 1 \mathrm{H})$.

7 - 1-(4-(isoquinolin-4-yl) piperazin-1-yl)-2-(1-methyl-1H-pyrazol-5-yl) ethanone. To a solution of 2-(1methyl-1H-pyrazol-5-yl) acetic acid (60 mg, $0.42 \mathrm{mmol}$ ), 4-(piperazin-1-yl)isoquinoline hydrochloride (110 $\mathrm{mg}, 0.42 \mathrm{mmol}$ ) and HATU (168 $\mathrm{mg}, 0.42 \mathrm{mmol})$ in DMF $(3 \mathrm{~mL})$ was added DIEA $(1.5 \mathrm{~mL})$ at room temperature. The reaction mixture was directly purified by Prep-HPLC under basic conditions to afford 1(4-(isoquinolin-4-yl) piperazin-1-yl)-2-(1-methyl-1H-pyrazol-5-yl) (54mg, 68\% yield, 100 purity) as a white solid. LC/MS C ${ }_{19} \mathrm{H}_{21} \mathrm{~N}_{5} \mathrm{O}[\mathrm{M}+\mathrm{H}]^{+}$calculated $\mathrm{m} / \mathrm{z}=336.17$; found $\mathrm{m} / \mathrm{z}=336.1 ; 1.60$ mins at $\lambda=254 \mathrm{~nm} .{ }^{1} \mathbf{H}-$ NMR $(400 \mathrm{MHz}, \mathrm{CDCl} 3) \delta(\mathrm{ppm})=9.02(\mathrm{~s}, 1 \mathrm{H}), 8.17(\mathrm{~s}, 1 \mathrm{H}), 8.09(\mathrm{~d}, J=8.4 \mathrm{~Hz}, 1 \mathrm{H}), 7.99(\mathrm{~d}, J=8.2 \mathrm{~Hz}, 1 \mathrm{H})$, $7.74(\mathrm{t}, J=7.6 \mathrm{~Hz}, 1 \mathrm{H}), 7.63(\mathrm{t}, J=7.6 \mathrm{~Hz}, 1 \mathrm{H}), 7.45(\mathrm{~s}, 1 \mathrm{H}), 6.14(\mathrm{~s}, 1 \mathrm{H}), 3.90(\mathrm{~s}, 5 \mathrm{H}), 3.83(\mathrm{~s}, 2 \mathrm{H}), 3.78(\mathrm{~s}$, $2 \mathrm{H}), 3.16(\mathrm{~s}, 4 \mathrm{H})$.

8 - 1-(4-(isoquinolin-4-yl) piperazin-1-yl)-2-(1-methyl-1H-pyrazol-3-yl) ethanone. To a solution of 2-(1methyl-1H-pyrazol-3-yl) acetic acid (70 mg, $0.49 \mathrm{mmol}$ ), 4-(piperazin-1-yl)isoquinoline hydrochloride (128 $\mathrm{mg}, 0.49 \mathrm{mmol})$ and HATU (196 $\mathrm{mg}, 0.49 \mathrm{mmol})$ in DMF $(3 \mathrm{~mL})$ was added DIEA $(1.5 \mathrm{~mL})$ at room temperature. The reaction mixture was directly purified by Prep-HPLC under basic conditions to afford 1(4-(isoquinolin-4-yl) piperazin-1-yl)-2-(1-methyl-1H-pyrazol-3-yl) (82mg, 87\% yield, 100 purity) as a white solid. LC/MS C ${ }_{19} \mathrm{H}_{21} \mathrm{~N}_{5} \mathrm{O}[\mathrm{M}+\mathrm{H}]^{+}$calculated $\mathrm{m} / z=336.17$; found $\mathrm{m} / \mathrm{z}=336.1 ; 1.74$ mins at $\lambda=254 \mathrm{~nm}$. ${ }^{1} \mathrm{H}-$ NMR $(400 \mathrm{MHz}, \mathrm{CDCl} 3) \delta(\mathrm{ppm})=8.99(\mathrm{~s}, 1 \mathrm{H}), 8.13(\mathrm{~s}, 1 \mathrm{H}), 8.09(\mathrm{~d}, J=8.4 \mathrm{~Hz}, 1 \mathrm{H}), 7.97(\mathrm{~d}, J=8.1 \mathrm{~Hz}, 1 \mathrm{H})$, $7.72(\mathrm{ddd}, J=8.4,6.9,1.3 \mathrm{~Hz}, 1 \mathrm{H}), 7.66-7.55(\mathrm{~m}, 1 \mathrm{H}), 7.31(\mathrm{~d}, J=2.1 \mathrm{~Hz}, 1 \mathrm{H}), 6.24(\mathrm{~d}, J=2.2 \mathrm{~Hz}, 1 \mathrm{H}), 4.12$ $-3.83(\mathrm{~m}, 7 \mathrm{H}), 3.83(\mathrm{~s}, 2 \mathrm{H}), 3.09(\mathrm{~d}, J=29.5 \mathrm{~Hz}, 4 \mathrm{H})$.

9 - 2-(1-isopropyl-1H-pyrazol-3-yl)-1-(4-(isoquinolin-4-yl)piperazin-1-yl)ethanone. To a solution of 2-(1isopropyl-1H-pyrazol-3-yl) acetic acid (30 mg, $0.16 \mathrm{mmol}), 4$-(piperazin-1-yl)isoquinoline hydrochloride (42 $\mathrm{mg}, 0.16 \mathrm{mmol})$ and HATU $(71 \mathrm{mg}, 0.16 \mathrm{mmol})$ in DMF $(3 \mathrm{~mL})$ was added DIEA $(1.5 \mathrm{~mL})$ at room temperature. The reaction mixture was directly purified by Prep-HPLC under basic conditions to afford 2(1-isopropyl-1H-pyrazol-3-yl)-1-(4-(isoquinolin-4-yl)piperazin-1-yl)ethanone (33 mg, 51\% yield, 100\% purity) as a white solid. $\mathbf{L C} / \mathbf{M S ~} \mathrm{C}_{21} \mathrm{H}_{25} \mathrm{~N}_{5} \mathrm{O}[\mathrm{M}+\mathrm{H}]^{+}$calculated $m / z=364.2 ;$ found $m / z=364.2 ; 1.72$ mins at 
$\lambda=254 \mathrm{~nm} .{ }^{1} \mathrm{H}-\mathrm{NMR}(400 \mathrm{MHz}, \mathrm{CDCl} 3) \delta(\mathrm{ppm})=8.99(\mathrm{~s}, 1 \mathrm{H}), 8.09(\mathrm{~d}, J=10.7 \mathrm{~Hz}, 2 \mathrm{H}), 7.97(\mathrm{~d}, J=8.1 \mathrm{~Hz}$, $1 \mathrm{H}), 7.71(\mathrm{dd}, J=11.3,4.1 \mathrm{~Hz}, 1 \mathrm{H}), 7.61(\mathrm{t}, J=7.2 \mathrm{~Hz}, 1 \mathrm{H}), 7.38(\mathrm{~d}, J=2.2 \mathrm{~Hz}, 1 \mathrm{H}), 6.24(\mathrm{~d}, J=2.2 \mathrm{~Hz}, 1 \mathrm{H})$, $4.45(\mathrm{dt}, J=13.4,6.7 \mathrm{~Hz}, 1 \mathrm{H}), 3.86(\mathrm{~d}, J=12.1 \mathrm{~Hz}, 6 \mathrm{H}), 3.05(\mathrm{~d}, J=41.2 \mathrm{~Hz}, 4 \mathrm{H}), 1.49(\mathrm{~d}, J=6.7 \mathrm{~Hz}, 6 \mathrm{H})$.

10 - 1-(4-(isoquinolin-4-yl) piperazin-1-yl)-2-(4-methylthiazol-2-yl) ethanone. To a solution of 2-(4methylthiazol-2-yl) acetic acid (111 mg, $0.60 \mathrm{mmol}$ ), 4-(piperazin-1-yl)isoquinoline hydrochloride (158 $\mathrm{mg}$, $0.60 \mathrm{mmol}$ ) and HATU $(250 \mathrm{mg}, 0.60 \mathrm{mmol})$ in DMF $(3 \mathrm{~mL})$ was added DIEA (1.5 mL) at room temperature. The reaction mixture was directly purified by Prep-HPLC under basic conditions to afford 1-(4-(isoquinolin4-yl) piperazin-1-yl)-2-(4-methylthiazol-2-yl) ethanone (28 mg, 20\% yield, $100 \%$ purity) as a white solid. . LC/MS C ${ }_{19} \mathrm{H}_{20} \mathrm{~N}_{4} \mathrm{OS}[\mathrm{M}+\mathrm{H}]^{+}$calculated $\mathrm{m} / z=353.14$; found $\mathrm{m} / \mathrm{z}=353.1 ; 1.77$ mins at $\lambda=254 \mathrm{~nm}$. ${ }^{1} \mathbf{H}$ NMR $\left(400 \mathrm{MHz}, \mathrm{CDCl}_{3}\right) \delta(\mathrm{ppm})=9.01(\mathrm{~s}, 1 \mathrm{H}), 8.16(\mathrm{~s}, 1 \mathrm{H}), 8.10(\mathrm{~d}, J=8.4 \mathrm{~Hz}, 1 \mathrm{H}), 7.98(\mathrm{~d}, J=8.2 \mathrm{~Hz}, 1 \mathrm{H}), 7.73$ $(\mathrm{t}, J=7.4 \mathrm{~Hz}, 1 \mathrm{H}), 7.62(\mathrm{t}, J=7.5 \mathrm{~Hz}, 1 \mathrm{H}), 6.86(\mathrm{~s}, 1 \mathrm{H}), 4.19(\mathrm{~s}, 2 \mathrm{H}), 3.89(\mathrm{~s}, 4 \mathrm{H}), 3.14(\mathrm{~s}, 4 \mathrm{H}), 2.44(\mathrm{~s}, 3 \mathrm{H})$.

11 - 1-(4-(isoquinolin-4-yl)piperazin-1-yl)-2-(4-(trifluoromethyl)thiazol-2-yl)ethan-1-one. To a flame dried vial, 4-(piperazin-1-yl)isoquinoline hydrochloride $\quad(50.0 \quad \mathrm{mg}, \quad 0.20 \mathrm{mmol}), \quad 2-(4-$ (trifluoromethyl)thiazol-2-yl)acetic acid $(50.7 \mathrm{mg}, 0.24 \mathrm{mmol})$, and HATU (114 $\mathrm{mg}, 0.30 \mathrm{mmol}$ ) was dissolved in anhydrous DMF $(2.00 \mathrm{~mL})$ under an argon atmosphere. Then triethylamine $(30.4 \mathrm{mg}, 42 \mu \mathrm{L}$, $0.30 \mathrm{mmol}$ ) was added and the reaction was left for 4 hours at room temperature. The reaction was quenched with ammonium chloride and extracted $3 x$ with $\mathrm{CH}_{2} \mathrm{Cl}_{2}$. The organic phases were combined, washed with sodium bicarbonate, brine, dried over magnesium sulfate, and concentrated in vacuo. The target compound was then isolated via HPLC under acidic conditions. 1-(4-(isoquinolin-4-yl)piperazin-1yl)-2-(4-(trifluoromethyl)thiazol-2-yl)ethan-1-one as a white solid (32 mg, 39\% yield, 99\% purity). LC/MS $\mathrm{C}_{19} \mathrm{H}_{17} \mathrm{~F}_{3} \mathrm{~N}_{4} \mathrm{OS}[\mathrm{M}+\mathrm{H}]^{+}$calculated $\mathrm{m} / \mathrm{z}=407.11$; found $\mathrm{m} / \mathrm{z}=407.1 ; 1.91 \mathrm{mins}$ at $\lambda=254 \mathrm{~nm}$. ${ }^{1} \mathrm{H}-\mathrm{NMR}$ $(\mathrm{CDCl}, 400 \mathrm{MHz}) \delta(\mathrm{ppm})=1.25-1.33(\mathrm{~m}, 1 \mathrm{H}) 3.12-3.31(\mathrm{~m}, 5 \mathrm{H}) 4.29(\mathrm{~s}, 2 \mathrm{H}) 7.62-7.69(\mathrm{~m}, 1 \mathrm{H}) 7.73$ - $7.84(\mathrm{~m}, 3 \mathrm{H}) 8.02(\mathrm{~d}, \mathrm{~J}=8.07 \mathrm{~Hz}, 1 \mathrm{H}) 8.13(\mathrm{~d}, \mathrm{~J}=8.31 \mathrm{~Hz}, 1 \mathrm{H}) 8.21(\mathrm{~s}, 1 \mathrm{H}) 9.05(\mathrm{~s}, 1 \mathrm{H})$

12 - 1-(4-(isoquinolin-4-yl) piperazin-1-yl)-2-(3-methylisoxazol-5-yl) ethanone. To a solution of 2-(3methylisoxazol-5-yl) acetic acid (50 mg, $0.35 \mathrm{mmol}$ ), 4-(piperazin-1-yl)isoquinoline hydrochloride $(97 \mathrm{mg}$, $0.35 \mathrm{mmol}$ ) and HATU (145 mg, $0.35 \mathrm{mmol})$ in DMF ( $3 \mathrm{~mL})$ was added DIEA (1.5 mL) at room temperature. The reaction mixture was directly purified by Prep-HPLC under basic conditions to afford 1-(4-(isoquinolin4-yl) piperazin-1-yl)-2-(3-methylisoxazol-5-yl) ethanone (12 mg, 10\% yield, 100\% purity) as a yellow solid. LC/MS C ${ }_{19} \mathrm{H}_{20} \mathrm{~N}_{4} \mathrm{O}_{2}[\mathrm{M}+\mathrm{H}]^{+}$calculated $\mathrm{m} / \mathrm{z}=337.16$; found $\mathrm{m} / \mathrm{z}=437.1 ; 1.75$ mins at $\lambda=254 \mathrm{~nm} .{ }^{1} \mathrm{H}-\mathrm{NMR}$ $\left(400 \mathrm{MHz}, \mathrm{CDCl}_{3}\right) \delta(\mathrm{ppm})=9.02(\mathrm{~s}, 1 \mathrm{H}), 8.18(\mathrm{~s}, 1 \mathrm{H}), 8.10(\mathrm{~d}, J=8.5 \mathrm{~Hz}, 1 \mathrm{H}), 7.99(\mathrm{~d}, J=8.1 \mathrm{~Hz}, 1 \mathrm{H}), 7.74$ (ddd, $J=8.3,6.9,1.3 \mathrm{~Hz}, 1 \mathrm{H}), 7.67-7.56(\mathrm{~m}, 1 \mathrm{H}), 6.13(\mathrm{~s}, 1 \mathrm{H}), 3.92(\mathrm{~s}, 4 \mathrm{H}), 3.87-3.76(\mathrm{~m}, 2 \mathrm{H}), 3.18(\mathrm{~d}, J$ $=4.7 \mathrm{~Hz}, 4 \mathrm{H}), 2.31(\mathrm{~s}, 3 \mathrm{H})$.

13 - 1-(4-(isoquinolin-4-yl) piperazin-1-yl)-2-(2-methyloxazol-4-yl) ethanone. To a solution of 2-(2methyloxazol-4-yl)acetic acid ( $80 \mathrm{mg}, 0.57 \mathrm{mmol}$ ) in DMF $(5 \mathrm{~mL}$ ) was added 4-(piperazin-1-yl)isoquinoline hydrochloride ( $170 \mathrm{mg}, 0.69 \mathrm{mmol}$ ) and HATU ( $325 \mathrm{mg}, 0.86 \mathrm{mmol}$ ) followed by DIEA ( $367 \mathrm{mg}, 2.85 \mathrm{mmol}$ ). The mixture was stirred at room temperature for 0.5 hour. Then the mixture was purified by Pre-HPLC under basic conditions to give desired 1-(4-(isoquinolin-4-yl) piperazin-1-yl)-2-(2-methyloxazol-4-yl) ethanone ( $36 \mathrm{mg}, 19 \%$ yield) as a white solid. $\mathrm{LC} / \mathrm{MS} \mathrm{C}_{19} \mathrm{H}_{20} \mathrm{~N}_{4} \mathrm{O}_{2}[\mathrm{M}+\mathrm{H}]^{+}$calculated $\mathrm{m} / \mathrm{z}=337.16$; found $\mathrm{m} / \mathrm{z}=337.0 ; 1.70 \mathrm{mins}$ at $\lambda=254 \mathrm{~nm} .{ }^{1} \mathrm{H}-\mathrm{NMR}\left(400 \mathrm{MHz}, \mathrm{CDCl}_{3}\right) \delta(\mathrm{ppm})=9.01(\mathrm{~s}, 1 \mathrm{H}), 8.18(\mathrm{~s}, 1 \mathrm{H}), 8.11$ 
(d, $J=7.8 \mathrm{~Hz}, 1 \mathrm{H}$ ), $7.99(\mathrm{~d}, J=8.1 \mathrm{~Hz}, 1 \mathrm{H}), 7.73$ (ddd, $J=8.3,6.9,1.3 \mathrm{~Hz}, 1 \mathrm{H}), 7.66-7.59(\mathrm{~m}, 1 \mathrm{H}), 7.56(\mathrm{~s}$, $1 \mathrm{H}), 3.88(\mathrm{~s}, 4 \mathrm{H}), 3.69(\mathrm{~d}, J=0.9 \mathrm{~Hz}, 2 \mathrm{H}), 3.24-3.09(\mathrm{~m}, 4 \mathrm{H}), 2.45(\mathrm{~s}, 3 \mathrm{H}) .{ }^{13} \mathrm{C}-\mathrm{NMR}\left(\mathrm{CDCl}_{3}, 100 \mathrm{MHz}\right) \delta$ $(\mathrm{ppm})=168.3,161.5,148.4,135.8,134.3,131.5,130.23,128.2,127.5,122.3,52.7,52.6,46.6,42.3,32.1$, 14.0. HRMS (ESI) $\mathrm{C}_{19} \mathrm{H}_{20} \mathrm{~N}_{4} \mathrm{O}_{2}[\mathrm{M}+\mathrm{Na}]^{+}$calculated $\mathrm{m} / \mathrm{z}=359.1484$; found $\mathrm{m} / \mathrm{z}=359.1474$

14 - 2-(2-cyclopropyloxazol-4-yl)-1-(4-(isoquinolin-4-yl)piperazin-1-yl)ethan-1-one. To a flame dried vial, 4-(piperazin-1-yl)isoquinoline hydrochloride $(50.0 \mathrm{mg}, 1 \mathrm{Eq}, 200 \mu \mathrm{mol}), 2$-(2-cyclopropyloxazol-4-yl)acetic acid $(40.2 \mathrm{mg}, 0.240 \mathrm{mmol})$, and HATU (114 mg, $0.30 \mathrm{mmol})$ was dissolved in anhydrous DMF (2.00 $\mathrm{mL})$ under an argon atmosphere. Then triethylamine $(30.4 \mathrm{mg}, 42 \mu \mathrm{L}, 0.30 \mathrm{mmol})$ was added and the reaction was left at 4 hours at room temperature. The reaction was monitored via LCMS. The reaction was quenched with sodium bicarbonate and extracted $3 x$ with $\mathrm{CH}_{2} \mathrm{Cl}_{2}$. The organic phases were combined, washed with brine, dried over magnesium sulfate, and concentrated in vacuo. The target compound was isolated via HPLC under acidic conditions. 2-(2-cyclopropyloxazol-4-yl)-1-(4-(isoquinolin-4-yl)piperazin-1yl)ethan-1-one ( $48 \mathrm{mg}, 66 \%$ yield, $99 \%$ purity) as a white solid. $\mathrm{LC} / \mathrm{MS} \mathrm{C}_{21} \mathrm{H}_{22} \mathrm{~N}_{4} \mathrm{O}_{2}[\mathrm{M}+\mathrm{H}]^{+}$calculated $\mathrm{m} / z$ $=363.16$; found $\mathrm{m} / \mathrm{z}=363.2 ; 1.76 \mathrm{mins}$ at $\lambda=254 \mathrm{~nm} .{ }^{1} \mathrm{H}-\mathrm{NMR}(\mathrm{CDCl} 3,400 \mathrm{MHz}) \delta(\mathrm{ppm})=1.00-1.06$ (m, 4 H) 1.60 (s, 2 H) 1.99 - 2.09 (m, 1 H) 3.09 - 3.23 (m, 4 H) 3.67 (s, 2 H) 3.88 (br. s., 2 H) 7.49 (s, 1 H) 7.59 - $7.66(\mathrm{~m}, 1 \mathrm{H}) 7.73(\mathrm{t}, \mathrm{J}=7.70 \mathrm{~Hz}, 1 \mathrm{H}) 7.98(\mathrm{~d}, \mathrm{~J}=8.31 \mathrm{~Hz}, 1 \mathrm{H}) 8.11(\mathrm{~d}, \mathrm{~J}=8.56 \mathrm{~Hz}, 1 \mathrm{H}) 8.18(\mathrm{~s}, 1 \mathrm{H}) 9.01$ (s, $1 \mathrm{H})$

15 - 2-(2-(tert-butyl)oxazol-4-yl)-1-(4-(isoquinolin-4-yl)piperazin-1-yl)ethan-1-one. To a flame dried vial, 4-(piperazin-1-yl)isoquinoline hydrochloride $(50.0 \mathrm{mg}, 0.20 \mathrm{mmol}), 2$-(2-(tert-butyl)oxazol-4-yl)acetic acid (44.0 mg, $0.24 \mathrm{mmol}$ ), and HATU (114 mg, $0.30 \mathrm{mmol}$ ) was dissolved in anhydrous DMF (2.0 mL) under an argon atmosphere. Then triethylamine $(30.4 \mathrm{mg}, 42 \mu \mathrm{L}, 0.30 \mathrm{mmol})$ was added and the reaction was left at 4 hours at room temperature. The reaction was monitored via LCMS. The reaction was quenched with sodium bicarbonate and extracted $3 x$ with $\mathrm{CH}_{2} \mathrm{Cl}_{2}$. The organic phases were combined, washed with brine, dried over magnesium sulfate, and concentrated in vacuo. The target compound was isolated via prep-HPLC under acidic conditions. 2-(2-(tert-butyl)oxazol-4-yl)-1-(4-(isoquinolin-4-yl)piperazin-1$\mathrm{yl}$ )ethan-1-one (36 mg, $48 \%$ yield, 99\% purity) as a white solid. LC/MS $\mathrm{C}_{22} \mathrm{H}_{26} \mathrm{~N}_{4} \mathrm{O}_{2}[\mathrm{M}+\mathrm{H}]^{+}$calculated $\mathrm{m} / \mathrm{z}$ $=379.21$; found $\mathrm{m} / \mathrm{z}=379.2 ; 1.829$ mins at $\lambda=254 \mathrm{~nm} .{ }^{1} \mathrm{H}-\mathrm{NMR}(\mathrm{CDCl} 3,400 \mathrm{MHz}) \delta(\mathrm{ppm})=1.37(\mathrm{~s}, 9 \mathrm{H})$ 3.17 (d, J=16.87 Hz, 4 H) 3.72 (s, 2 H) 3.92 (br. s., 4 H) $7.56-7.67(\mathrm{~m}, 2 \mathrm{H}) 7.75$ (t, J=7.70 Hz, 1 H) 7.99 (d, $\mathrm{J}=8.07 \mathrm{~Hz}, 1 \mathrm{H}) 8.09-8.20(\mathrm{~m}, 2 \mathrm{H}) 9.02(\mathrm{~s}, 1 \mathrm{H})$

General synthetic route towards the synthesis of compounds $15-23$ with 17 as a representative example:

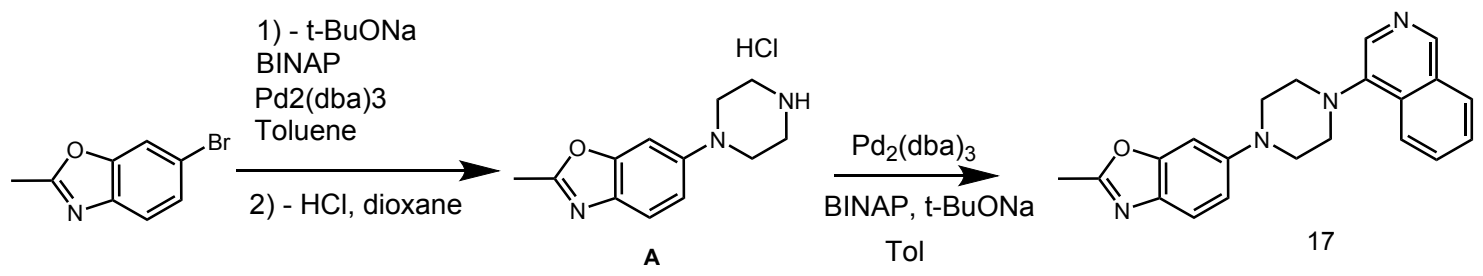

A - 2-methyl-6-(piperazin-1-yl)benzo[d]oxazole hydrochloride. To a flame dried vial, 6-bromo-2methylbenzo[d]oxazole $(50.0 \mathrm{mg}, 0.24 \mathrm{mmol})$, tert-butyl piperazine-1-carboxylate $(48.3 \mathrm{mg}, 0.26 \mathrm{mmol})$ , 2,2'-bis(diphenylphosphaneyl)-1,1'-binaphthalene (7.34, $0.012 \mathrm{mmol}$ ), $\mathrm{Pd}_{2} \mathrm{dba}_{3}(5.40 \mathrm{mg}, 0.059 \mathrm{mmol}$ ), 
and sodium 2-methylpropan-2-olate $(45.3 \mathrm{mg}, 0.47 \mathrm{mmol})$ were dissolved in Toluene $(2.36 \mathrm{~mL})$ under an argon atmosphere. The solution was stirred at $80^{\circ} \mathrm{C}$ for 3 hours and monitored via LCMS. The reaction was filtered through celite and the boc-protected compound was isolated via by column chromatography (silica gel, $\mathrm{CH} 2 \mathrm{Cl} 2$ : $\left.\mathrm{MeOH}\left(0.1 \% \mathrm{NH}_{3}\right)=9: 1\right)$. The clean fractions were collected, combined, and concentrated. The afforded solid was then subjected to hydrogen chloride ( $8.60 \mathrm{mg}, 3 \mathrm{~mL}, 1 \mathrm{Eq}, 236 \mu \mathrm{mol})$ (4M in Dioxane). Once complete, the solvent was removed yielding a target compound clean enough for advancement. 2-methyl-6-(piperazin-1-yl)benzo[d]oxazole hydrochloride (32 mg, 53\% yield, 95\% purity). LC/MS C ${ }_{12} \mathrm{H}_{15} \mathrm{NO}_{3}[\mathrm{M}+\mathrm{H}]^{+}$calculated $m / z=218.12$; found $\mathrm{m} / z=218.1 ; 0.56$ mins at $\lambda=254 \mathrm{~nm}$.

17 - 6-(4-(isoquinolin-4-yl)piperazin-1-yl)-2-methylbenzo[d]oxazole. To a flame dried vial, 2-methyl-6(piperazin-1-yl)benzo[d]oxazole hydrochloride $(50.0 \mathrm{mg}, 0.20 \mathrm{mmol})$, 4-bromoisoquinoline (41.0 mg, 0.20 $\mathrm{mmol}$ ), Tris(dibenzylideneacetone)dipalladium(0) (4.51 mg, $0.49 \mathrm{mmol}), 2,2$ '-bis(diphenylphosphaneyl)1,1'-binaphthalene $(6.14 \mathrm{mg}, 0.10 \mathrm{mmol})$ and sodium 2-methylpropan-2-olate $(37.9 \mathrm{mg}, 0.39 \mathrm{mmol})$ were dissolved in Toluene $\left(2.36 \mathrm{~mL}\right.$ ) under an argon atmosphere. The solution was stirred at $110^{\circ} \mathrm{C}$ for 4 hours and monitored via LCMS. The reaction was filtered through celite and the target compound was isolated by column chromatography (silica gel, $\mathrm{CH} 2 \mathrm{Cl} 2$ : $\left.\mathrm{MeOH}\left(0.1 \% \mathrm{NH}_{3}\right)=9: 1\right)$. The clean fractions were collected, combined and concentrated. 6-(4-(isoquinolin-4-yl)piperazin-1-yl)-2-methylbenzo[d]oxazole as a white powder (11 mg, $16 \%$ yield, $90 \%$ purity). LC/MS $\mathrm{C}_{21} \mathrm{H}_{20} \mathrm{~N}_{4} \mathrm{O}[\mathrm{M}+\mathrm{H}]^{+}$calculated $\mathrm{m} / \mathrm{z}=345.16$; found $\mathrm{m} / \mathrm{z}=345.0 ; 1.8 \mathrm{mins}$ at $\lambda=254 \mathrm{~nm} .{ }^{1} \mathrm{H}-\mathrm{NMR}(\mathrm{CDCl} 3,400 \mathrm{MHz}) \delta(\mathrm{ppm})=2.64(\mathrm{~s}, 3 \mathrm{H}) 3.41(\mathrm{~d}, \mathrm{~J}=4.65 \mathrm{~Hz}$, 4 H) 3.47 - $3.57(\mathrm{~m}, 4 \mathrm{H}) 7.07$ (d, J=8.80 Hz, 1 H) $7.13(\mathrm{~s}, 1 \mathrm{H}) 7.57$ (d, J=8.80 Hz, 1 H) 7.65 (t, J=7.46 Hz, 1 H) 7.76 (t, J=7.58 Hz, 1 H) 8.02 (d, J=8.07 Hz, 1 H) 8.19 (d, J=8.56 Hz, 1 H) 8.29 (br. s., 1 H) 9.05 (br. s., 1 H).

16 - 5-(4-(isoquinolin-4-yl)piperazin-1-yl)-2-methylbenzo[d]oxazole. 2-methyl-5-(piperazin-1yl)benzo[d]oxazole hydrochloride (128 mg, $0.50 \mathrm{mmol}$ ) 4-bromoisoquinoline (105 mg, $0.50 \mathrm{mmol}$ ) sodium tert-butoxide (72.7 mg, $0.76 \mathrm{mmol}$ ) (R)-2,2'-bis(diphenylphosphaneyl)-1,1'-binaphthalene (31.4 mg, 0.05 $\mathrm{mmol}$ ) were heated in dry Toluene $(2.5 \mathrm{~mL})$ for three hours at 140 degrees. The reaction was then filtered through celite and concentrated under reduced pressure. The crude was then purified by column chromatography (silica gel, $\mathrm{CH} 2 \mathrm{Cl} 2: \mathrm{MeOH}\left(0.1 \% \mathrm{NH}_{3}\right)=9: 1$ ). 5-(4-(isoquinolin-4-yl)piperazin-1-yl)-2methylbenzo[d]oxazole as a white powder (100 mg, 58\% yield, 94\% purity). LC/MS $\mathrm{C}_{21} \mathrm{H}_{20} \mathrm{~N}_{4} \mathrm{O}[\mathrm{M}+\mathrm{H}]^{+}$ calculated $m / z=345.16$; found $m / z=345.2 ; 1.93$ mins at $\lambda=254 \mathrm{~nm} .{ }^{1} \mathbf{H}-\mathbf{N M R}\left(\mathrm{CDCl}_{3}, 400 \mathrm{MHz}\right) \delta(\mathrm{ppm})$ $=9.05(\mathrm{~s}, 1 \mathrm{H}), 8.29(\mathrm{~s}, 1 \mathrm{H}), 8.20(\mathrm{~d}, 1 \mathrm{H}, \mathrm{J}=8.4 \mathrm{~Hz}), 8.03(\mathrm{~d}, 1 \mathrm{H}, \mathrm{J}=8.4 \mathrm{~Hz}), 7.80-7.76(\mathrm{~m}, 1 \mathrm{H}), 7.66(\mathrm{dd}, 1 \mathrm{H}$, $\mathrm{J}=7.2 \mathrm{~Hz}), 7.67(\mathrm{~d}, 1 \mathrm{H}, \mathrm{J}=8.8 \mathrm{~Hz}), 7.14(\mathrm{~d}, 1 \mathrm{H}, \mathrm{J}=2.4 \mathrm{~Hz}), 7.09-7.06(\mathrm{dd}, 1 \mathrm{H}, \mathrm{J} 1=8.4 \mathrm{~Hz}, \mathrm{~J} 2=2.0 \mathrm{~Hz}), 3.51-$ $3.41(\mathrm{~m}, 8 \mathrm{H}, 2.64(\mathrm{~s}, 3 \mathrm{H})$.

18 - 4-(4-(2-methyl-2H-indazol-6-yl)piperazin-1-yl)isoquinoline. To a flame dried vial, 1-methyl-6(piperazin-1-yl)-1H-indazole hydrochloride ( $60 \mathrm{mg}, 0.24 \mathrm{mmol}$ ), 4-bromoisoquinoline ( $54 \mathrm{mg}, 0.26 \mathrm{mmol}$ ), Tris(dibenzylideneacetone)dipalladium(0) $(5.4 \mathrm{mg}, 5.9 \mu \mathrm{mol}), \quad 2,2$ '-bis(diphenylphosphaneyl)-1,1'binaphthalene ( $7.4 \mathrm{mg}, 12 \mu \mathrm{mol}$ ) and sodium 2-methylpropan-2-olate ( $23 \mathrm{mg}, 0.24 \mathrm{mmol}$ ) were dissolved in Toluene $(2.4 \mathrm{~mL})$ under an argon atmosphere. The solution was stirred at $110^{\circ} \mathrm{C}$ for 3 hours and monitored via LCMS. The reaction was filtered through celite and the target compound was isolated via by column chromatography (silica gel, $\mathrm{CH} 2 \mathrm{Cl} 2$ : $\left.\mathrm{MeOH}\left(0.1 \% \mathrm{NH}_{3}\right)=9: 1\right)$. 4-(4-(2-methyl-2H-indazol-6- 
yl)piperazin-1-yl)isoquinoline as a white powder (30 mg, 36\% yield, 98\% purity). LC/MS $\mathrm{C}_{21} \mathrm{H}_{21} \mathrm{~N}_{4}[\mathrm{M}+\mathrm{H}]^{+}$ calculated $\mathrm{m} / \mathrm{z}=344.18$; found $\mathrm{m} / \mathrm{z}=344.1 ; 1.771 \mathrm{mins}$ at $\lambda=254 \mathrm{~nm} .{ }^{1} \mathbf{H}-\mathbf{N M R}\left(\mathrm{DMSO}_{-} \mathrm{d}_{6}, 400 \mathrm{MHz}\right) \delta$ $(\mathrm{ppm})=9.06(\mathrm{~s}, 1 \mathrm{H}), 8.26(\mathrm{~s}, 1 \mathrm{H}), 8.18-8.12(\mathrm{~m}, 2 \mathrm{H}), 7.84\left(\mathrm{dd}, 1 \mathrm{H}, \mathrm{J}_{1}=\mathrm{J}_{2}=8.4 \mathrm{~Hz}\right), 7.71\left(\mathrm{dd}, 1 \mathrm{H}, \mathrm{J}_{1}=\mathrm{J}_{2}=\right.$ $8.0 \mathrm{~Hz}$ ), $7.56(\mathrm{~d}, 1 \mathrm{H}, \mathrm{J}=9.2 \mathrm{~Hz}), 7.03-7.01\left(\mathrm{dd}, 1 \mathrm{H}, \mathrm{J}_{1}=9.2 \mathrm{~Hz}, \mathrm{~J}_{2}=2.0 \mathrm{~Hz}\right), 4.10(\mathrm{~s}, 3 \mathrm{H}), 3.43(\mathrm{bs}, 4 \mathrm{H}), 3.30$ (bs, 4H). ${ }^{13}$ C-NMR (DMSO-d $\left.6,100 \mathrm{MHz}\right) \delta(\mathrm{ppm})=150.0,149.9,148.1,143.2,132.9,130.9,130.5,129.3$, $128.5,127.8,124.5,122.9,121.0,117.3,117.0,99.2,52.8,50.3$. HRMS (ESI) $\mathrm{C}_{21} \mathrm{H}_{21} \mathrm{~N}_{5}[\mathrm{M}+\mathrm{H}]^{+}$calculated $m / z=344.1875 ;$ found $m / z=344.1877$

19 - 4-(4-(2-methyl-2H-pyrazolo[3,4-b]pyridin-6-yl)piperazin-1-yl)isoquinoline. To a dried vial, 4(piperazin-1-yl)isoquinoline hydrochloride $(100 \mathrm{mg}, 0.40 \mathrm{mmol})$, 6-chloro-2-methyl-2H-pyrazolo[3,4b]pyridine ( $80.5 \mathrm{mg}, 0.48 \mathrm{mmol}$ ), dicyclohexyl(2,6-diisopropoxy-[1,1'-biphenyl]-3-yl)phosphane (9.34 mg, $20.0 \mu \mathrm{mol})$, sodium 2-methylpropan-2-olate ( $115 \mathrm{mg}, 1.20 \mathrm{mmol}$ ) and RuPhos Pd G3 (8.37 mg, $10.0 \mu \mathrm{mol})$ were dissolved in Tetrahydrofuran $(6 \mathrm{~mL})$ under an argon atmosphere. The solution was stirred at $80^{\circ} \mathrm{C}$ for 4 hours and monitored via LCMS. LCMS suggested that the reaction was proceeding slowly, thus another equivalent of dicyclohexyl(2,6-diisopropoxy-[1,1'-biphenyl]-3-yl)phosphane (9.34 mg, $20.0 \mu \mathrm{mol}$ ) and RuPhos Pd G3 $(8.37 \mathrm{mg}, 10.0 \mu \mathrm{mol})$ were added and allowed to react overnight. The reaction was filtered through celite and the compound was isolated via column chromatography (silica gel, $\mathrm{CH} 2 \mathrm{Cl} 2$ : $\left.\operatorname{MeOH}\left(0.1 \% \mathrm{NH}_{3}\right)=9: 1\right)$. 4-(4-(2-methyl-2H-pyrazolo[3,4-b]pyridin-6-yl)piperazin-1-yl)isoquinoline as a light orange crystalline solid ( $26 \mathrm{mg}, 18 \%$ yield, $98 \%$ purity). $\mathrm{LC} / \mathrm{MS}_{20} \mathrm{H}_{20} \mathrm{~N}_{6}[\mathrm{M}+\mathrm{H}]^{+}$calculated $\mathrm{m} / z=$ 345.17; found $\mathrm{m} / \mathrm{z}=345.2 ; 1.85 \mathrm{mins}$ at $\lambda=254 \mathrm{~nm} .{ }^{1} \mathrm{H}-\mathrm{NMR}(\mathrm{CDCl} 3,400 \mathrm{MHz}) \delta(\mathrm{ppm})=9.06(\mathrm{~s}, 1 \mathrm{H})$ $8.24(\mathrm{~s}, 1 \mathrm{H}), 8.22-8.19(\mathrm{~d}, \mathrm{~J}=8.4,1 \mathrm{H}), 8.15-8.13(\mathrm{~d}, \mathrm{~J}=8.12,1 \mathrm{H}), 7.96-7.93(\mathrm{~d}, \mathrm{~J}=8.88 .1 \mathrm{H}), 7.85-7.82$ $(\mathrm{m}, 2 \mathrm{H}), 7.73-7.70(\mathrm{t}, \mathrm{J}=7.4,1 \mathrm{H}), 6.91-6.89(\mathrm{~d}, \mathrm{~J}=8.92,1 \mathrm{H}), 3.95(\mathrm{~s}, 4 \mathrm{H}), 3.34(\mathrm{~s}, 3 \mathrm{H}), 3.26(\mathrm{~s}, 4 \mathrm{H})$

20 - 4-(4-(7-fluoro-2-methyl-2H-indazol-6-yl)piperazin-1-yl)isoquinoline. To a flame dried vial, 7-fluoro2-methyl-6-(piperidin-4-yl)-2H-indazole hydrochloride $(50.0 \mathrm{mg}, 0.19 \mathrm{mmol})$, 4-bromoisoquinoline (38.6 $\mathrm{mg}, \quad 0.19 \mathrm{mmol}), \quad$ Tris(dibenzylideneacetone)dipalladium(0) $(4.24 \mathrm{mg}, \quad 4.63 \mu \mathrm{mol}), \quad 2,2^{\prime}-$ bis(diphenylphosphaneyl)-1,1'-binaphthalene (5.77 mg, $9.27 \mu \mathrm{mol}$ ) and sodium 2-methylpropan-2-olate (35.6 mg, $0.37 \mathrm{mmol}$ ) were dissolved in Toluene $(1.85 \mathrm{~mL}$ ) under an argon atmosphere. The solution was stirred at $110^{\circ} \mathrm{C}$ for 3 hours and monitored via LCMS. The reaction was filtered through celite and the target compound was isolated via column chromatography (silica gel, $\mathrm{CH} 2 \mathrm{Cl} 2$ : $\left.\mathrm{MeOH}\left(0.1 \% \mathrm{NH}_{3}\right)=9: 1\right)$. 4(4-(7-fluoro-2-methyl-2H-indazol-6-yl)piperazin-1-yl)isoquinoline as a light yellow crystalline solid (49 mg, 73\% yield, 98\% purity). $\mathrm{LC} / \mathrm{MS} \mathrm{C}_{21} \mathrm{H}_{21} \mathrm{FN}_{5}[\mathrm{M}+\mathrm{H}]^{+}$calculated $\mathrm{m} / \mathrm{z}=362.17$; found $\mathrm{m} / \mathrm{z}=362.1 ; 1.80$ mins at $\lambda=254 \mathrm{~nm} .{ }^{1} \mathrm{H}-\mathrm{NMR}(\mathrm{CDCl} 3,400 \mathrm{MHz}) \delta(\mathrm{ppm})=3.31(\mathrm{~d}, \mathrm{~J}=4.65 \mathrm{~Hz}, 4 \mathrm{H}) 3.36-3.45(\mathrm{~m}, 4 \mathrm{H}) 4.16(\mathrm{~s}, 3$ H) 7.05 (dd, J=8.93, $7.21 \mathrm{~Hz}, 1 \mathrm{H}) 7.49$ (d, J=8.80Hz, $1 \mathrm{H}) 7.67-7.73(\mathrm{~m}, 1 \mathrm{H}) 7.82$ (ddd, J=8.44, 6.97, 1.22 $\mathrm{Hz}, 1 \mathrm{H}) 8.17(\mathrm{~d}, \mathrm{~J}=8.56 \mathrm{~Hz}, 1 \mathrm{H}) 8.13(\mathrm{~d}, \mathrm{~J}=7.83 \mathrm{~Hz}, 1 \mathrm{H}) 8.27(\mathrm{~s}, 1 \mathrm{H}) 8.37$ (d, J=2.69) Hz, 1 H) $9.05(\mathrm{~s}, 1 \mathrm{H})$. ${ }^{13} \mathrm{C}-\mathrm{NMR}\left(\mathrm{CDCl}_{3}, 100 \mathrm{MHz}\right) \delta(\mathrm{ppm})=148.1,145.4,143.4,142.9,141.4,141.3,134.2,134.1,132.9,131.5$, 129.8, 129.3, 128.0, 127.2, 124.1, 124.0, 122.6, 121.5, 121.4, 117.6, 115.8, 115.7, 53.1, 51.7, 51.6, 40.5. HRMS (ESI) $\mathrm{C}_{21} \mathrm{H}_{20} \mathrm{FN}_{5}[\mathrm{M}+\mathrm{H}]^{+}$calculated $m / z=362.1781$; found $m / z=362.1763$

21 - 4-(4-(1-methyl-1H-indazol-6-yl)piperazin-1-yl)isoquinoline. To a flame dried vial, 1-methyl-6(piperazin-1-yl)-1H-indazole hydrochloride (60 mg, $0.24 \mathrm{mmol}$ ), 4-bromoisoquinoline ( $54 \mathrm{mg}, 0.26 \mathrm{mmol}$ ), 
Tris(dibenzylideneacetone)dipalladium(0) (5.4 mg, 5.9 umol), 2,2'-bis(diphenylphosphaneyl)-1,1'binaphthalene $(7.4 \mathrm{mg}, 12 \mu \mathrm{mol}$ ) and sodium 2-methylpropan-2-olate $(23 \mathrm{mg}, 0.24 \mathrm{mmol})$ were dissolved in Toluene $(2.4 \mathrm{~mL})$ under an argon atmosphere. The solution was stirred at $100^{\circ} \mathrm{C}$ for 3 hours and monitored via LCMS. The reaction was filtered through celite and the target compound was isolated via column chromatography (silica gel, $\mathrm{CH} 2 \mathrm{Cl} 2$ : $\left.\mathrm{MeOH}\left(0.1 \% \mathrm{NH}_{3}\right)=9: 1\right)$. 4-(4-(1-methyl-1H-indazol-6yl)piperazin-1-yl)isoquinoline as a light yellow crystalline solid (20 mg, 24\% yield, 98\% purity). LC/MS $\mathrm{C}_{21} \mathrm{H}_{21} \mathrm{~N}_{4}[\mathrm{M}+\mathrm{H}]^{+}$calculated $\mathrm{m} / \mathrm{z}=344.18$; found $\mathrm{m} / \mathrm{z}=344.1 ; 1.97$ mins at $\lambda=254 \mathrm{~nm}$. ${ }^{1} \mathrm{H}-\mathrm{NMR}(\mathrm{CDCl} 3$, $400 \mathrm{MHz}) \delta(\mathrm{ppm})=3.43(\mathrm{t}, \mathrm{J}=4.28 \mathrm{~Hz}, 4 \mathrm{H}) 3.58$ (br. s., $4 \mathrm{H}) 4.06(\mathrm{~s}, 3 \mathrm{H}) 6.81(\mathrm{~s}, 1 \mathrm{H}) 7.03(\mathrm{~d}, \mathrm{~J}=8.80 \mathrm{~Hz}, 1$ H) $7.61-7.68(\mathrm{~m}, 2 \mathrm{H}) 7.72-7.79(\mathrm{~m}, 1 \mathrm{H}) 7.89(\mathrm{~s}, 1 \mathrm{H}) 8.02(\mathrm{~d}, \mathrm{~J}=8.07 \mathrm{~Hz}, 1 \mathrm{H}) 8.21(\mathrm{~d}, \mathrm{~J}=8.31 \mathrm{~Hz}, 1 \mathrm{H}) 8.31$ $(\mathrm{s}, 1 \mathrm{H}) 9.05(\mathrm{~s}, 1 \mathrm{H}) .{ }^{13} \mathrm{C}-\mathrm{NMR}\left(\mathrm{CDCl}_{3}, 100 \mathrm{MHz}\right) \delta(\mathrm{ppm})=150.9,147.4,143.7,141.2,132.6,131.8,131.5$, $130.5,129.2,128.4,127.6,122.6,121.5,118.6,114.1,93.7,52.7,50.5,35.4$. HRMS (ESI) $\mathrm{C}_{21} \mathrm{H}_{21} \mathrm{~N}_{5}[\mathrm{M}+\mathrm{H}]^{+}$ calculated $m / z=344.1875$; found $m / z=344.1864$

22 - 4-(4-(2-methyl-[1,2,4]triazolo[1,5-a]pyridin-7-yl)piperazin-1-yl)isoquinoline. To a dried vial, 4(piperazin-1-yl)isoquinoline hydrochloride $(50 \mathrm{mg}, 0.20 \mathrm{mmol}$ ), 7-bromo-2-methyl-[1,2,4]triazolo[1,5a]pyridine (42 mg, $0.20 \mathrm{mmol}$ ), dicyclohexyl(2,6-diisopropoxy-[1,1'-biphenyl]-3-yl)phosphane (4.7 mg, 10 $\mu \mathrm{mol}$ ), sodium 2-methylpropan-2-olate ( $38 \mathrm{mg}, 0.40 \mathrm{mmol}$ ) and RuPhos Pd G3 (4.2 mg, $5.0 \mu \mathrm{mol}$ ) were dissolved in THF $(2 \mathrm{~mL})$ under an argon atmosphere. The solution was stirred at $80^{\circ} \mathrm{C}$ for 4 hours and monitored via LCMS. The reaction was filtered through celite and the compound was isolated via column chromatography (silica gel, $\mathrm{CH} 2 \mathrm{Cl} 2: \mathrm{MeOH}\left(0.1 \% \mathrm{NH}_{3}\right)=9: 1$ ) as an amber solid. 4-(4-(2-methyl$[1,2,4]$ triazolo[1,5-a]pyridin-7-yl)piperazin-1-yl)isoquinoline as a white powder (10 $\mathrm{mg}, 15 \%$ yield, $98 \%$ purity LC/MS $\mathrm{C}_{20} \mathrm{H}_{20} \mathrm{~N}_{6}[\mathrm{M}+\mathrm{H}]^{+}$calculated $\mathrm{m} / z=345.18$; found $\mathrm{m} / z=345.2 ; 1.53$ mins at $\lambda=254 \mathrm{~nm}$. ${ }^{1} \mathrm{H}-$ NMR $(C D C l 3,400 \mathrm{MHz}) \delta(\mathrm{ppm})=9.06(\mathrm{~s}, 1 \mathrm{H}), 8.31-8.27(\mathrm{~m}, 2 \mathrm{H}), 8.19-8.17(\mathrm{~d}, \mathrm{~J}=8.4,1 \mathrm{H}), 8.04-8.02(\mathrm{~d}$, $\mathrm{J}=8.12,1 \mathrm{H})$ 7.80-7.76 (t, J = 7.16, $1 \mathrm{H}), 7.69-7.65(\mathrm{t}, \mathrm{J}=7.48,1 \mathrm{H}), 6.90(\mathrm{~s}, 1 \mathrm{H}), 6.79-6.77(\mathrm{~d}, \mathrm{~J}=7.56,1$ H), $3.63(\mathrm{~s}, 4 \mathrm{H}) 3.40-3.39(\mathrm{~m}, 4 \mathrm{H}), 2.57(\mathrm{~s}, 3 \mathrm{H})$.

23 - 4-(4-(2-methylimidazo[1,2-a]pyridin-7-yl)piperazin-1-yl)isoquinoline. To a flame dried vial, 2methyl-7-(piperazin-1-yl)imidazo[1,2-a]pyridine hydrochloride $(50.0 \mathrm{mg}, 0.20 \mu \mathrm{mol}), 4$-bromoisoquinoline (49.4 mg, $0.237 \mu \mathrm{mol})$, sodium 2-methylpropan-2-olate $\left(\begin{array}{llll}28.5 & \mathrm{mg}, 0.30 \mu \mathrm{mol}\end{array}\right), \quad 2,2^{\prime}-$ bis(diphenylphosphaneyl)-1,1'-binaphthalene $(6.16 \mathrm{mg}, 9.89 \mu \mathrm{mol})$, and $\mathrm{Pd}_{2} \mathrm{dba}_{3}(45 \mathrm{mg}, 0.05 \mathrm{mmol}$ ) were dissolved in Toluene $\left(1.98 \mathrm{~mL}\right.$ ) under an argon atmosphere. The solution was stirred at $110^{\circ} \mathrm{C}$ for 3 hours and monitored via LCMS. The reaction was filtered through celite and the target compound was isolated via HPLC under acidic conditions. 4-(4-(2-methylimidazo[1,2-a]pyridin-7-yl)piperazin-1-yl)isoquinoline as a white powder (20 mg, $29 \%$ yield, $98 \%$ purity $\mathrm{LC} / \mathrm{MS} \mathrm{C}_{21} \mathrm{H}_{25} \mathrm{~N}_{5}[\mathrm{M}+\mathrm{H}]^{+}$calculated $\mathrm{m} / \mathrm{z}=344.18$; found $\mathrm{m} / \mathrm{z}$ $=344.1 ; 1.73$ mins at $\lambda=254 \mathrm{~nm} .{ }^{1} \mathrm{H}-\mathrm{NMR}(\mathrm{CDCl} 3,400 \mathrm{MHz}) \delta(\mathrm{ppm})=2.23$ (s, $\left.3 \mathrm{H}\right) 3.28$ (br. s., $\left.4 \mathrm{H}\right) 3.47$ (br. s., 4 H) 6.65 (s, 1 H) 6.84 (d, J=7.34 Hz, 1 H) 7.37 (s, 1 H) 7.68 (s, 1H) 7.80 (s, 1 H) 8.14 (d, J=8.31 Hz, 1 H) $8.11(\mathrm{~d}, \mathrm{~J}=8.56 \mathrm{~Hz}, 1 \mathrm{H}) 8.21-8.26(\mathrm{~m}, 2 \mathrm{H}) 9.04(\mathrm{~s}, 1 \mathrm{H})$.

Synthetic route towards compound 24: 


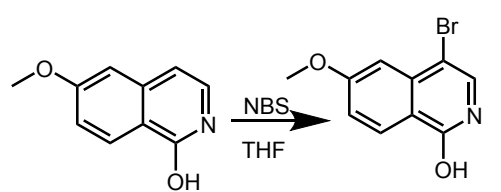

A

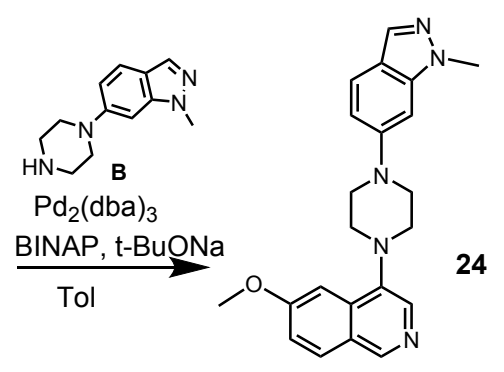

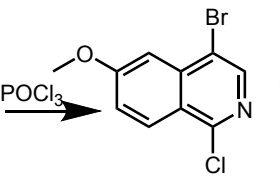

B<smiles>CC(C)(C)Oc1ccc2cncc(Br)c2c1</smiles>

C

A - 4-bromo-6-methoxyisoquinolin-1-ol. To a solution of 6-methoxyisoquinolin-1-ol (300 mg, $1.71 \mathrm{mmol}$ ) in THF (15 mL) was added N-Bromosuccinimide $(335 \mathrm{mg}, 1.88 \mathrm{mmol})$. The mixture was stirred at room temperature for 3 hours. The reaction was concentrated in vacuo and purified by column chromatography (silica gel, Ethyl acetate : Petroleum ether $=30: 70$ ) to give 4-bromo-6-methoxyisoquinolin-1-ol A (440 mg, 93\% yield, $90 \%$ purity) as a white solid. $\mathbf{L C} / \mathrm{MS} \mathrm{C}_{10} \mathrm{H}_{8} \mathrm{BrNO}_{2}[\mathrm{M}+\mathrm{H}]^{+}$calculated $\mathrm{m} / \mathrm{z}=353.97$; found $\mathrm{m} / \mathrm{z}=$ $354.1 ; 1.73$ mins at $\lambda=254 \mathrm{~nm}$.

B - 4-bromo-1-chloro-6-methoxyisoquinoline. 4-bromo-6-methoxyisoquinolin-1-ol (440 mg, $1.73 \mathrm{mmol}$ ) was dissolved in $\mathrm{POCl}_{3}(10 \mathrm{~mL})$. The mixture was stirred at $100^{\circ} \mathrm{C}$ for 2 hours. The solvent was removed under reduced pressure and the crude was diluted in ethyl acetate. The mixture was neutralized with $\mathrm{NaOH}(\mathrm{aq})$ at $0{ }^{\circ} \mathrm{C}$ and was further extracted with Ethyl Acetate $(150 \mathrm{~mL} \times 3)$. The combined organic layers were dried over anhydrous $\mathrm{Na}_{2} \mathrm{SO}_{4}$ and concentrated in vacuo to give 4-bromo-1-chloro-6methoxyisoquinoline ( $260 \mathrm{mg}, 55.2 \%$ yield, $80 \%$ purity) as a yellow solid. LC/MS $\mathrm{C}_{10} \mathrm{H}_{8} \mathrm{BrClNO}_{2}[\mathrm{M}+\mathrm{H}]^{+}$ calculated $m / z=271.94 ;$ found $m / z=272.1 ; 2.21$ mins at $\lambda=254 \mathrm{~nm}$.

C - 4-bromoisoquinolin-6-ol. Red phosphorus ( $446 \mathrm{mg}, 14.39 \mathrm{mmol}$ ) was added to the solution of 4-bromo1-chloro-6-methoxyisoquinoline $(260 \mathrm{mg}, 0.95 \mathrm{mmol}$ ) in hydroiodic acid ( $3 \mathrm{~mL}, 57 \mathrm{wt} . \%$ in water). The mixture was stirred at $140^{\circ} \mathrm{C}$ for 3 hours. After cooling to room temperature, the mixture was poured into saturated sodium bicarbonate. The mixture was extracted with $\mathrm{CH}_{2} \mathrm{Cl}_{2}(120 \mathrm{~mL} \times 3)$. The combined organic layers were dried over anhydrous $\mathrm{Na}_{2} \mathrm{SO}_{4}$ and concentrated in vacuo to get 4-bromoisoquinolin-6-ol (100 $\mathrm{mg}, 46.7 \%$ yield, $90 \%$ purity) as a yellow solid. $\mathrm{LC} / \mathrm{MS} \mathrm{C}_{10} \mathrm{H}_{8} \mathrm{BrNO}_{2}[\mathrm{M}+\mathrm{H}]^{+}$calculated $\mathrm{m} / z=222.96$; found $m / z=224.0 ; 1.69$ mins at $\lambda=254 \mathrm{~nm}$.

D - 4-bromo-6-methoxyisoquinoline. To a solution of 4-bromoisoquinolin-6-ol ( $100 \mathrm{mg}, 0.45 \mathrm{mmol}$ ) in DMF $(10 \mathrm{~mL})$ was added $\mathrm{K}_{2} \mathrm{CO}_{3}(62 \mathrm{mg}, 0.45 \mathrm{mmol})$ followed by $\mathrm{CH}_{3} \mathrm{l}(64 \mathrm{mg}, 0.45 \mathrm{mmol})$. The mixture was stirred at room temperature for 3 hours. Then ethyl acetate $(30 \mathrm{~mL})$ was added and the mixture was washed with water (10 mL x3). The organic layer was dried over anhydrous $\mathrm{Na}_{2} \mathrm{SO}_{4}$ and concentrated in vacuo to give 4-bromo-6-methoxyisoquinoline (45 mg, 42.4\% yield, 95\% purity) as yellow solid. LC/MS $\mathrm{C}_{10} \mathrm{H}_{8} \mathrm{BrNO}_{2}$ $[\mathrm{M}+\mathrm{H}]^{+}$calculated $m / z=237.98 ;$ found $m / z=238.0 ; 1.78$ mins at $\lambda=254 \mathrm{~nm}$. 
24 - 6-methoxy-4-(4-(1-methyl-1H-indazol-6-yl)piperazin-1-yl)isoquinoline. To a solution of 4-bromo-6methoxyisoquinoline ( $45 \mathrm{mg}, 0.19 \mathrm{mmol})$ in toluene $(10 \mathrm{~mL}), 1$-methyl-6-(piperazin-1-yl)-1H-indazole (60 $\mathrm{mg}, 0.28 \mathrm{mmol}$ ), t-BuONa (55 mg, $0.57 \mathrm{mmol}$ ), BINAP (12 mg, $0.02 \mathrm{mmol}$ ) and Pd2(dba)3 (9 mg, 0.01 $\mathrm{mmol}$ ) were added. Under $\mathrm{N} 2$ atmosphere, the mixture was stirred at $100^{\circ} \mathrm{C}$ for 2 hours. Then the reaction was concentrated in vacuo and purified by pre-HPLC under basic conditions to afford 6-methoxy-4-(4-(1methyl-1H-indazol-6-yl)piperazin-1-yl)isoquinoline (0.9 $\mathrm{mg}, 1.3 \%$ yield, $97 \%$ purity) as a white solid. . LC/MS C ${ }_{22} \mathrm{H}_{23} \mathrm{~N}_{5} \mathrm{O}[\mathrm{M}+\mathrm{H}]^{+}$calculated $m / z=374.19 ;$ found $\mathrm{m} / \mathrm{z}=274.0 ; 2.03 \mathrm{mins}$ at $\lambda=254 \mathrm{~nm}$. ${ }^{1} \mathrm{H}-\mathrm{NMR}$ $(400 \mathrm{MHz}, \mathrm{CDCl} 3) \delta(\mathrm{ppm})=8.92(\mathrm{~s}, 1 \mathrm{H}), 8.24(\mathrm{~s}, 1 \mathrm{H}), 7.91(\mathrm{~d}, J=8.9 \mathrm{~Hz}, 1 \mathrm{H}), 7.87(\mathrm{~s}, 1 \mathrm{H}), 7.63(\mathrm{~d}, J=9.2 \mathrm{~Hz}$, $1 \mathrm{H}), 7.44(\mathrm{~s}, 1 \mathrm{H}), 7.01(\mathrm{~d}, J=7.1 \mathrm{~Hz}, 2 \mathrm{H}), 6.78(\mathrm{~s}, 1 \mathrm{H}), 4.04(\mathrm{~s}, 3 \mathrm{H}), 3.99(\mathrm{~s}, 3 \mathrm{H}), 3.56(\mathrm{~s}, 4 \mathrm{H}), 3.39(\mathrm{~s}, 4 \mathrm{H})$.

Synthetic route towards the synthesis of compound 25 :

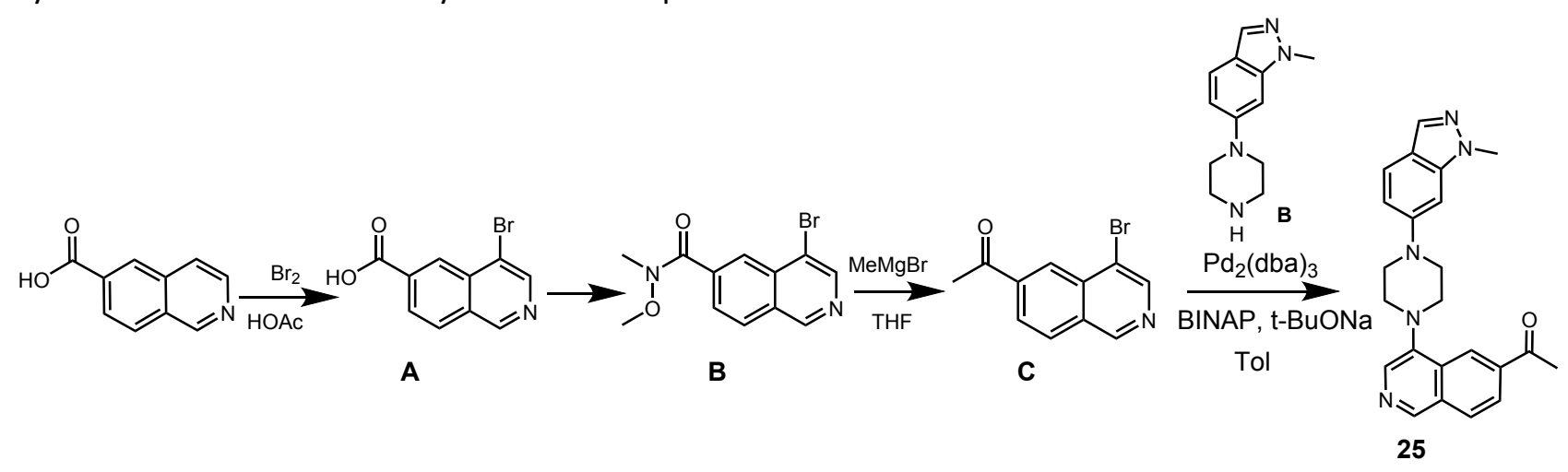

A - To a solution of isoquinoline-6-carboxylic acid ( $600 \mathrm{mg}, 3.47 \mathrm{mmol}$ ) in $\mathrm{AcOH}(15 \mathrm{~mL})$ was slowly added $\mathrm{Br}_{2}(1.1 \mathrm{~g}, 6.94 \mathrm{mmol})$ in $5 \mathrm{~mL} \mathrm{AcOH}$. The reaction mixture was heated at $100^{\circ} \mathrm{C}$ for 24 hours. After cooling to room temperature, the solvent was removed under reduced pressure. $\mathrm{H}_{2} \mathrm{O}(20 \mathrm{~mL})$ and $\mathrm{MeOH}(10 \mathrm{~mL})$ were added and the suspension was stirred for $10 \mathrm{~min}$, filtered and dried under vacuum to afford 4bromoisoquinoline-6-carboxylic acid ( $650 \mathrm{mg}$, $45 \%$ yield, $68 \%$ purity) as a yellow solid. LC/MS $\mathrm{C}_{10} \mathrm{H}_{6} \mathrm{BrN}_{5} \mathrm{O}$ $[\mathrm{M}+\mathrm{H}]^{+}$calculated $\mathrm{m} / \mathrm{z}=251.96$; found $\mathrm{m} / \mathrm{z}=252.1 ; 2.19$ mins at $\lambda=254 \mathrm{~nm}$.

B - To a solution of 4-bromoisoquinoline-6-carboxylic acid (300 mg, $1.195 \mathrm{mmol}$ ) and N,Odimethylhydroxylamine hydrochloride $(232 \mathrm{mg}, 2.39 \mathrm{mmol})$, HATU(545 mg, $1.43 \mathrm{mmol})$ in DMF(10 mL) was added DIPEA $(2 \mathrm{~mL})$. The reaction mixture was stirred at room temperature overnight. $\mathrm{H}_{2} \mathrm{O}(10 \mathrm{~mL})$ was then added and the mixture was extracted with EtOAc $(150 \mathrm{~mL} \times 3)$, washed with brine, dried over magnesium sulfate and concentrated. The crude was purified by column chromatography (silica gel, Ethyl acetate : Petroleum ether $=25: 75)$ to afford 4-bromo- $\mathrm{N}$-methoxy- $\mathrm{N}$-methylisoquinoline-6-carboxamide (225 mg, 64\% yield) as a colorless oil. $\mathbf{L C} / \mathbf{M S ~ C}_{12} \mathrm{H}_{11} \mathrm{BrN}_{2} \mathrm{O}_{2}[\mathrm{M}+\mathrm{H}]^{+}$calculated $\mathrm{m} / \mathrm{z}=295.00$; found $\mathrm{m} / \mathrm{z}=$ $295.1 ; 1.80$ mins at $\lambda=254 \mathrm{~nm}$

$\mathrm{C}$ - Under $\mathrm{N} 2$, at $-78{ }^{\circ} \mathrm{C}$, to a solution of 4-bromo-N-methoxy- $\mathrm{N}$-methylisoquinoline-6-carboxamide (190 $\mathrm{mg}, 0.646 \mathrm{mmol})$ in THF $(15 \mathrm{~mL})$ was added $\mathrm{MeMgBr}(0.26 \mathrm{~mL}, 0.776 \mathrm{mmol})$. The reaction mixture was stirred at room temperature for 2 hours. $\mathrm{NH}_{4} \mathrm{Cl}$ (aq., $100 \mathrm{~mL}$ ) was added and the reaction stirred for $5 \mathrm{~min}$. The mixture was then extracted with EtOAc $(150 \mathrm{~mL} \times 3)$, washed with brine, dried over magnesium sulfate and concentrated. The crude was purified by column chromatography (silica gel, Ethyl acetate : Petroleum 
ether $=50: 50)$ to give 1-(4-bromoisoquinolin-6-yl)ethanone $(120 \mathrm{mg}, 75 \%$ yield, $97 \%$ purity) as a white solid. LC/MS $\mathrm{C}_{11} \mathrm{H}_{8} \mathrm{BrNO}[\mathrm{M}+\mathrm{H}]^{+}$calculated $m / z=249.98$; found $m / z=250.0 ; 1.74$ mins at $\lambda=254 \mathrm{~nm}$.

25 - 1-(4-(4-(1-methyl-1H-indazol-6-yl) piperazin-1-yl) isoquinolin-6-yl) ethanone. To a solution of 1methyl-6-(piperazin-1-yl)-1H-indazole B (90 mg, $0.42 \mathrm{mmol}$ ) in toluene $(10 \mathrm{~mL}), 1$-(4-bromoisoquinolin-6yl)ethanone (70 mg, $0.28 \mathrm{mmol}$ ), t-BuONa ( $81 \mathrm{mg}, 0.84 \mathrm{mmol}$ ), BINAP (17 mg, $0.03 \mathrm{mmol}$ ) and Pd2(dba)3 (13 mg, $0.014 \mathrm{mmol}$ ) were added. Under N2 atmosphere, the mixture was stirred at $100^{\circ} \mathrm{C}$ for 3 hours. The reaction was concentrated and purified by prep-HPLC under basic conditions to give 1-(4-(4-(1methyl-1H-indazol-6-yl) piperazin-1-yl) isoquinolin-6-yl) ethanone (12 mg, 11\% yield, 1005 purity) as a yellow solid. $\mathrm{LC} / \mathrm{MS} \mathrm{C}_{23} \mathrm{H}_{23} \mathrm{~N}_{5} \mathrm{O}[\mathrm{M}+\mathrm{H}]^{+}$calculated $\mathrm{m} / \mathrm{z}=386.19$; found $\mathrm{m} / \mathrm{z}=386.0 ; 1.97 \mathrm{mins}$ at $\lambda=254$ nm. ${ }^{1} \mathrm{H}-\mathrm{NMR}(400 \mathrm{MHz}, \mathrm{CDCl} 3) \delta(\mathrm{ppm})=9.08(\mathrm{~s}, 1 \mathrm{H}), 8.81(\mathrm{~s}, 1 \mathrm{H}), 8.37(\mathrm{~s}, 1 \mathrm{H}), 8.18-8.13(\mathrm{~m}, 1 \mathrm{H}), 8.07$ $(\mathrm{d}, J=8.6 \mathrm{~Hz}, 1 \mathrm{H}), 7.87(\mathrm{~s}, 1 \mathrm{H}), 7.64(\mathrm{~d}, J=8.8 \mathrm{~Hz}, 1 \mathrm{H}), 7.01(\mathrm{dd}, J=8.9,2.0 \mathrm{~Hz}, 1 \mathrm{H}), 6.81(\mathrm{~s}, 1 \mathrm{H}), 4.04(\mathrm{~d}, J$ $=10.8 \mathrm{~Hz}, 3 \mathrm{H}), 3.60(\mathrm{~s}, 4 \mathrm{H}), 3.50-3.38(\mathrm{~m}, 4 \mathrm{H}), 2.74(\mathrm{~d}, J=22.9 \mathrm{~Hz}, 3 \mathrm{H}) .{ }^{13} \mathrm{C}-\mathrm{NMR}$ (DMSO-d, $\left.100 \mathrm{MHz}\right) \delta$ $(\mathrm{ppm})=198.4,150.7,147.8,144.1,141.6,137.5,134.0,132.4,130.6,130.3,129.1,125.7,124.3,121.4$, 118.1, 113.8, 94.0, 52.9, 49.9, 35.6, 27.5. HRMS (ESI) $\mathrm{C}_{23} \mathrm{H}_{23} \mathrm{~N}_{5} \mathrm{O}[\mathrm{M}+\mathrm{Na}]^{+}$calculated $\mathrm{m} / \mathrm{z}=386.1981$; found $m / z=386.1985$

26 - 4-(4-(1-methyl-1H-indazol-6-yl)piperazin-1-yl)isoquinoline-6-carbonitrile. To a flame dried vial, 4(piperazin-1-yl)isoquinoline-6-carbonitrile hydrochloride $(50.00 \mathrm{mg}, 1.8 \mathrm{~mL}, 0.18 \mathrm{mmol}$ ), 6-bromo-1methyl-1H-indazole ( $42.25 \mathrm{mg}, 0.20 \mathrm{mmol}$ ), dicyclohexyl(2,6-diisopropoxy-[1,1'-biphenyl]-3-yl)phosphane (4.246 mg, $9.1 \mu \mathrm{mol})$, sodium 2-methylpropan-2-olate $(34.98 \mathrm{mg}, 0.36 \mathrm{mmol}$ ) , and RuPhos (3.805 mg, 4.6 $\mu \mathrm{mol})$ were dissolved in Toluene $(5.34 \mathrm{~mL})$ under an argon atmosphere. The solution was stirred at $110^{\circ}$ $\mathrm{C}$ for 4 hours and monitored via LCMS. The reaction was filtered through celite and the target compound was isolated by column chromatography (silica gel, $\left.\mathrm{CH} 2 \mathrm{Cl} 2: \mathrm{MeOH}\left(0.1 \% \mathrm{NH}_{3}\right)=9: 1\right)$. 4-(4-(1-methyl-1Hindazol-6-yl)piperazin-1-yl)isoquinoline-6-carbonitrile isoquinoline as a white powder (30 mg, 45\% yield, 98\% purity). LC/MS $\mathrm{C}_{22} \mathrm{H}_{20} \mathrm{~N}_{6}[\mathrm{M}+\mathrm{H}]^{+}$calculated $\mathrm{m} / \mathrm{z}=369.19$; found $\mathrm{m} / \mathrm{z}=369.1 ; 1.86$ mins at $\lambda=254$ nm. ${ }^{1} \mathrm{H}-\mathrm{NMR}(\mathrm{CDCl}, 400 \mathrm{MHz}) \delta(\mathrm{ppm})=3.37$ - 3.47 (m, $\left.4 \mathrm{H}\right) 3.59$ (br. s., $\left.4 \mathrm{H}\right) 4.07$ (s, $\left.3 \mathrm{H}\right) 6.84(\mathrm{~s}, 1 \mathrm{H})$ $7.03(\mathrm{~d}, \mathrm{~J}=8.80 \mathrm{~Hz}, 1 \mathrm{H}) 7.66(\mathrm{~d}, \mathrm{~J}=8.80 \mathrm{~Hz}, 1 \mathrm{H}) 7.80$ (d, J=8.56 Hz, $1 \mathrm{H}) 7.89(\mathrm{~s}, 1 \mathrm{H}) 8.12(\mathrm{~d}, \mathrm{~J}=8.56 \mathrm{~Hz}, 1 \mathrm{H})$ $8.44(\mathrm{~s}, 1 \mathrm{H}) 8.59(\mathrm{~s}, 1 \mathrm{H}) 9.11(\mathrm{~s}, 1 \mathrm{H}) .{ }^{13} \mathrm{C}-\mathrm{NMR}\left(\mathrm{CDCl}_{3}, 100 \mathrm{MHz}\right) \delta(\mathrm{ppm})=147.8,143.2,141.2,134.7$, $132.6,130.7,129.7,129.4,129.1,128.0,121.6,118.8,118.6,114.3,113.4,94.15,52.9,50.7,35.4$. HRMS (ESI) $\mathrm{C}_{22} \mathrm{H}_{20} \mathrm{~N}_{6}[\mathrm{M}+\mathrm{Na}]^{+}$calculated $\mathrm{m} / \mathrm{z}=391.1647$; found $\mathrm{m} / \mathrm{z}=391.1632$

Synthetic route towards the synthesis of compound 27 :

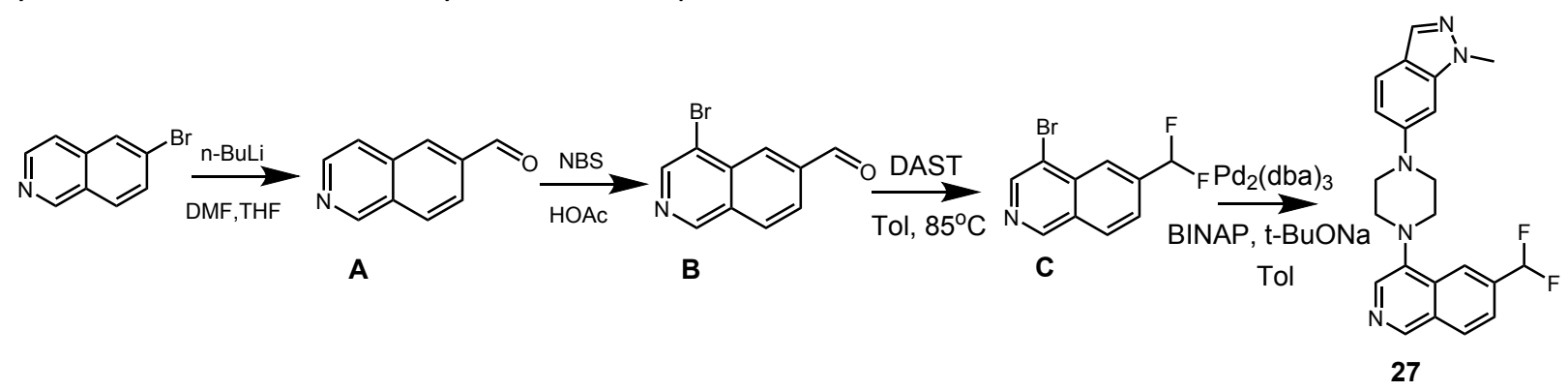

A - isoquinoline-6-carbaldehyde. 6-bromoisoquinoline $(3 \mathrm{~g}, 14.42 \mathrm{mmol})$ in anhydrous THF (20 $\mathrm{mL})$ was cooled to $-78^{\circ} \mathrm{C}$. n-BuLi $(1.2 \mathrm{~g}, 18.75 \mathrm{mmol})$ was added slowly over 10 minutes and the mixture was stirred 
for 30 minutes at $-78^{\circ} \mathrm{C}$. Then anhydrous DMF $(1.26 \mathrm{~g}, 17.30 \mathrm{mmol})$ was added slowly at $-78^{\circ} \mathrm{C}$ and the mixture was stirred at room temperature for 3 hours. LCMS monitored the reaction. The reaction was quenched with ammonium chloride $(50 \mathrm{~mL})$ and the solution was extracted with Ethyl Acetate $(150 \mathrm{~mL}$ $\mathrm{x} 3$ ). The organic layers were combined, dried over magnesium sulfate and concentrated. The crude was purified by column chromatography (silica gel, Ethyl acetate : Petroleum ether $=25: 75$ ). Isoquinoline-6carbaldehyde (600mg, $27 \%$ yield, $96 \%$ purity) as a white solid. $\mathbf{L C} / \mathrm{MS} \mathrm{C}_{10} \mathrm{H}_{7} \mathrm{NO}[\mathrm{M}+\mathrm{H}]^{+}$calculated $\mathrm{m} / z=$ 158.05 ; found $m / z=158.0 ; 1.66$ mins at $\lambda=254 \mathrm{~nm}$.

B - 4-bromoisoquinoline-6-carbaldehyde. To a solution of isoquinoline-6-carbaldehyde $(600 \mathrm{mg}, 3.8$ $\mathrm{mmol})$ in $\mathrm{AcOH}(15 \mathrm{~mL})$ was added NBS $(744 \mathrm{mg}, 4.2 \mathrm{mmol})$. The reaction was stirred at $90^{\circ} \mathrm{C}$ for 3 hours. The solvent was then removed under reduced pressure. The crude was purified by column chromatography (silica gel, Ethyl acetate : Petroleum ether $=40: 60$ ) to afford the 4-bromoisoquinoline-6carbaldehyde (374 mg, $42 \%$ yield, $100 \%$ purity) as a white solid. $\mathrm{LC} / \mathrm{MS} \mathrm{C}_{10} \mathrm{H}_{6} \mathrm{BrNO}[\mathrm{M}+\mathrm{H}]^{+}$calculated $\mathrm{m} / z$ $=235.96$; found $\mathrm{m} / \mathrm{z}=236.0 ; 1.89$ mins at $\lambda=254 \mathrm{~nm}$.

C - 4-bromo-6-(difluoromethyl)isoquinoline. Under $\mathrm{N}_{2}$, at $0^{\circ} \mathrm{C}$, to a solution of 4-bromoisoquinoline-6carbaldehyde $(130 \mathrm{mg}, 0.55 \mathrm{mmol})$ in anhydrous toluene $(15 \mathrm{~mL})$ was added DAST $(1.215 \mathrm{~g}, 5.5 \mathrm{mmol})$ slowly. The mixture was heated at $85^{\circ} \mathrm{C}$ for 2 hours. The solution was then poured in cold $\mathrm{NaHCO}(\mathrm{aq})$ and extracted with Ethyl Acetate $(150 \mathrm{~mL} \times 3)$, washed with brine, dried over magnesium sulfate and concentrated to get the crude product which was purified by column chromatography (silica gel, Ethyl acetate : Petroleum ether $=20: 80)$. 4-bromo-6-(difluoromethyl)isoquinoline $(110 \mathrm{mg}, 78 \%$ yield, $90 \%$ purity) as a white solid. $\mathbf{L C} / \mathrm{MS} \mathrm{C}_{10} \mathrm{H}_{6} \mathrm{BrF}_{2} \mathrm{~N}[\mathrm{M}+\mathrm{H}]^{+}$calculated $\mathrm{m} / \mathrm{z}=257.97$; found $\mathrm{m} / \mathrm{z}=256.0 ; 1.89$ mins at $\lambda=254 \mathrm{~nm}$.

27 - 6-(difluoromethyl)-4-(4-(1-methyl-1H-indazol-6-yl) piperazin-1-yl) isoquinoline. Under $\mathrm{N}_{2}$, to a solution of 4-bromo-6-(difluoromethyl)isoquinoline (100 mg,0.39 mmol), 1-methyl-6-(piperazin-1-yl)-1Hindazole (102 mg, $0.47 \mathrm{mmol}$ ) and t-BuONa (112 mg,1.17 mmol) in Tol(10mL) was added Pd2(dba)3 (36 $\mathrm{mg}, 0.039 \mathrm{mmol}$ ) and BINAP $\left(73 \mathrm{mg}, 0.117 \mathrm{mmol}\right.$ ). The reaction was stirred at $100^{\circ} \mathrm{C}$ for 2 hours. The reaction was then filtered through celite and the solvent was removed under reduced pressure. The crude was purified by Prep-HPLC under basic conditions to afford the 6-(difluoromethyl)-4-(4-(1-methyl-1Hindazol-6-yl) piperazin-1-yl) isoquinoline (64 mg, $42 \%$ yield, 100\% purity) as a white solid. LC/MS $\mathrm{C}_{22} \mathrm{H}_{21} \mathrm{~F}_{2} \mathrm{~N}_{5}[\mathrm{M}+\mathrm{H}]^{+}$calculated $\mathrm{m} / z=394.1$; found $\mathrm{m} / \mathrm{z}=394.1 ; 2.06$ mins at $\lambda=254 \mathrm{~nm}$. ${ }^{1} \mathbf{H}-\mathrm{NMR}$ (400 $\mathrm{MHz}, \mathrm{CDCl} 3) \delta(\mathrm{ppm})=9.08(\mathrm{~s}, 1 \mathrm{H}), 8.37(\mathrm{~s}, 1 \mathrm{H}), 8.33(\mathrm{~s}, 1 \mathrm{H}), 8.10(\mathrm{~d}, J=8.6 \mathrm{~Hz}, 1 \mathrm{H}), 7.87(\mathrm{~s}, 1 \mathrm{H}), 7.75(\mathrm{~d}$, $J=8.5 \mathrm{~Hz}, 1 \mathrm{H}), 7.63(\mathrm{~d}, J=8.9 \mathrm{~Hz}, 1 \mathrm{H}), 7.05-6.70(\mathrm{~m}, 3 \mathrm{H}), 4.05(\mathrm{~s}, 3 \mathrm{H}), 3.57(\mathrm{~s}, 4 \mathrm{H}), 3.44-3.38(\mathrm{~m}, 4 \mathrm{H})$. ${ }^{13} \mathrm{C}-\mathrm{NMR}\left(\mathrm{CDCl}_{3}, 100 \mathrm{MHz}\right) \delta(\mathrm{ppm})=150.9,148.0,143.6,141.2,135.7,135.4,135.2,133.9,132.6,131.7$, $129.9,129.1,123.7-123.6\left(t, J_{C-F}=4.8 \mathrm{~Hz}\right), 121.6,120.6-120.4(t, 7.6 \mathrm{~Hz}), 118.7,116.8,114.5,114.1,112.1$, 93.8, 52.9, 50.5, 35.4. HRMS (ESI) $\mathrm{C}_{22} \mathrm{H}_{21} \mathrm{~F}_{2} \mathrm{~N}_{5}[\mathrm{M}+\mathrm{H}]^{+}$calculated $\mathrm{m} / z=394.1843$; found $\mathrm{m} / \mathrm{z}=394.1850$ 